\title{
Explaining Universality*
}

\section{Infinite Limit Systems in the Renormalization Group Method}

\author{
Jingyi $\mathrm{Wu}^{\dagger}$ \\ October 7, 2021 \\ Forthcoming in Synthese \\ Please cite the published version
}

\begin{abstract}
I analyze the role of infinite idealizations used in the renormalization group (RG hereafter) method in explaining universality across microscopically different physical systems in critical phenomena. I argue that despite the reference to infinite limit systems such as systems with infinite correlation lengths during the RG process, the key to explaining universality in critical phenomena need not involve infinite limit systems. I develop my argument by introducing what I regard as the explanatorily relevant property in RG explanations: linearization* property; I then motivate and prove a proposition about the linearization* property in support of my view. As a result, infinite limit systems in RG explanations are dispensable.
\end{abstract}

\section{Introduction}

Universality in critical phenomena refers to the almost identical macroscopic behaviors manifested by microscopically different physical systems near critical phase transitions. ${ }^{1}$ Critical

*Acknowledgements: I'm extremely grateful to Ben Feintzeig, Kareem Khalifa, John Norton, Patricia Palacios, Laura Ruetsche, Jim Weatherall, and two anonymous reviewers for their invaluable comments on previous drafts. Thanks to Sorin Bangu, Bob Batterman, Liam Kofi Bright, and Kade Cicchella, audience members at the Foundations of Physics 2018 Conference in Utrecht, audience members at the MCMP, and members of the Philosophy of Physics Research Group at UC Irvine for helpful discussions on the topic. I'm grateful to David for his years of companionship and support. This paper was made possible in part through the support of grant \#61048 from the John Templeton Foundation. The opinions expressed in this publication are those of the author and do not necessarily reflect the views of the John Templeton Foundation.

†Department of Logic and Philosophy of Science, UC Irvine. Jingyi.Wu@uci.edu

${ }^{1}$ There are examples of universality that do not concern critical phase transitions, for instance when we utilize the same equation to calculate the periods of pendulums made of different materials (see Batterman (2001) for more details). This paper only concerns universal behaviors near critical phase transitions. 
phase transitions are certain transformations in the phenomenological features of physical systems, often characterized by the second-order discontinuities of some parameters of the systems. In explaining universality using arguably our best methods to date, the renormalization group (RG hereafter) methods, we make use of the thermodynamic limit by taking the number of particles to infinity. ${ }^{2}$ Many argue that we should understand thermodynamic limits in the RG method as infinite idealizations, because only systems with an infinite number of particles can explain universality exhibited by real systems (Kadanoff, 2000; Batterman, 2005; Morrison, 2015). Having indispensable infinite idealizations in one's explanation comes with epistemic implications. For one thing, one might think that systems with an infinite number of particles are fictitious. To interpret infinite limits in the RG method as infinite idealizations would contradict many prominent accounts of scientific explanation (such as Hempel and Oppenheim (1948); Strevens (2008); Craver (2006); Woodward (2005)), which require that the explanans has to at least be approximately true to provide an explanation for the target explanandum. ${ }^{3}$ Moreover, infinite idealizations may engender paradoxes (see Shech (2013); Palacios and Valente (2021)) or hinder certain reductionist theses (see Batterman (2001); Palacios (2019)). ${ }^{4}$

On the other hand, numerous authors argue that the distinction between infinite limit systems and finite systems is not at all pronounced, and as a result, RG methods' role in explaining universality does not force us to accept all the implications that come with infinite idealizations (Butterfield, 2011; Norton, 2012; Callender, 2001; Palacios, 2019). Butterfield, for example, maintains that the singularity of infinite limit systems could be smoothly approached by finite systems, and therefore the status of infinite limit systems is not entirely

\footnotetext{
${ }^{2}$ Two notes are in order. First, as will become clear in later sections, the RG methods are arguably the best because they explain universality by showing that the microscopic details that differentiate systems are irrelevant and they provide a sophisticated way of calculating the critical exponents. Second, later in the paper I will identify another less noted use of infinity in the RG method: the RG iterations limit (see Palacios (2019) for further discussions). For our current purposes, the use of thermodynamic limits already points to the core tension with respect to the role of infinite idealizations.

${ }^{3}$ There is a separate debate about whether RG explanations, regardless of whether it involves infinite idealizations, fit into any of the above listed accounts of explanations. Many maintain that RG explanations constitute a special kind of non-causal explanations, though they disagree as to exactly what kind they are (see, for instance, Batterman (2000); Batterman and Rice (2014); Reutlinger (2014); Khalifa et al. (2020)). Note also that some accounts of explanations allow for fictional explanans (Bokulich, 2008, 2012).

${ }^{4}$ Thanks an anonymous referee for suggesting this way of framing the issue. See also Shech (2018) and Fletcher et al. (2019) for an overview of philosophical issues concerning infinite idealizations.
} 
special (Butterfield, 2011). However, these remarks have not put the debate to rest. Batterman further argues in recent papers that infinite limit systems are essential in RG explanations because the RG trajectories of infinite systems flow to the nontrivial fixed point but those of finite systems do not (Batterman, 2017, 2019). Morrison $(2015,110)$ argues that infinite limit systems in the RG method are the only means possible for calculating values of critical exponents. ${ }^{5}$ Following Shech (2018), I call those who believe that only infinite limit systems can explain universality essentialists, and those who reject this view dispensabilists, so named because they maintain that infinite limit systems are dispensable. ${ }^{6}$

The difficulty around analyzing the use of infinite idealizations in the explanation of universality in critical phenomena is twofold. First, the essentialists are often vague about whether the extra properties that infinite idealizations exhibit, for example the flow to the nontrivial fixed point, are truly indispensable to the explanation of universality. Second, the dispensabilists are often vague about exactly how finite systems, or properties pertaining to finite systems, can provide explanations of our target phenomena and render the infinite idealizations dispensable.

This paper aims to fill this vacuum. Here, I will advance a dispensabilist's view, and suggest that in RG explanations, the explanatorily relevant functions purportedly only exhibited by infinite limit systems can be adequately, if not better, provided using large but finite systems. ${ }^{7}$ In doing so, I meet the critics at their strongest points. In response to Batterman (2017, 2019), I argue that his candidate property of the infinite limit systems, what I call the convergence property, is not indispensable for the explanations. In response to Morrison (2015), I point out that a property of the finite systems, what I call the linearization* property, can adequately recover the values of critical exponents without loss.

In Section 2, I will present a philosophical framework following Norton (2012)'s heuristic

\footnotetext{
${ }^{5}$ The relevant concepts such as "flow," "fixed point," and "RG trajectories" will be introduced in Section 3.2.

${ }^{6}$ While Shech (2018) intends his distinction to extend to other scientific accounts such as understanding, representation, and prediction, I focus on explanation here.

${ }^{7}$ In this paper I do not go into details about whether RG methods, regardless of the use of infinite limit systems, provide an explanation for universality, and exactly what account of explanation it is. For a cautious reader who does not agree that RG methods provide an explanation for universality at all, she is encouraged to read my claim in the following way: if infinite limit systems provide an explanation of universality in RG methods, then we have reasons to think that large but finite systems do as well.
} 
that in my view captures the central disagreements between the two sides. In Section 3.1, I will identify the explanandum that I am interested in; in Section 3.2, I will introduce the RG method and sketch the role of infinite limits in the RG process; in Section 3.3, I will present Batterman's defense of infinite limit systems in RG explanations. According to Batterman, infinite limit systems are indispensable precisely because infinite limit systems possess convergence properties, which are absent in finite systems. In Section 4, I will respond to Batterman by pointing out that the convergence property he regards as essential in RG explanations is not as explanatorily relevant as the linearization* property, which is defined in connection to the linearization* process crucial in the RG method. Finally, in Section 5, I will present both heuristic and technical evidence inspired by Wilson and Kogut (1974) and Yin (2011) to motivate and prove a proposition demonstrating that, under reasonable conditions, linearization* properties and convergence properties provide the same explanatory functions in terms of demarcating the universality classes. Because points with linearization* properties can be finite systems and linearization* properties have additional explanatory merits, I maintain my position that in RG explanations, infinite limit systems are dispensable, if the conditions are met. This paper is accompanied by a three-part techinical appendix. Appendix A contains a brief technical overview of the linearization* process, Appendix B contains the proofs of propositions, and Appendix C contains a glossary of technical terms.

\section{Explanatorily Relevant Properties}

In situations of scientific explanation where the explanans putatively involves infinite limit systems, how do we determine whether the infinite systems are essential in the explanation? Norton (2012) suggests that we need to focus on the comparison between certain properties of finite and infinite systems. In particular, we compare certain property of the infinite limit system with the corresponding limiting property of finite systems-the limit of a sequence of the corresponding properties of finite systems as we take a given parameter to infinity. Accordign to Norton, if the properties of infinite limit systems agree with the limiting properties of finite systems, then infinite limit systems do not perform anything that finite systems cannot 


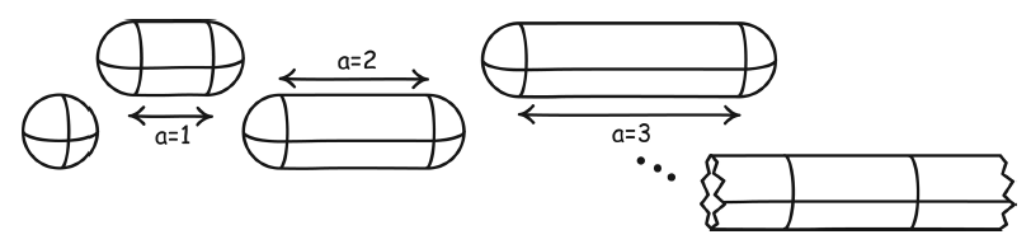

Figure 1: Unit sphere elongated into a sequence of capsules. When the property we compare is the ratio of surface area to volume, the property of the limit system agrees with the limiting property of sequence of capsules. (Norton, 2012)

do regarding these properties, and are thus dispensable. In what follows, I discuss and offer an amendment to Norton's heuristic. I suggest that infinite limit systems are indispensable in scientific explanations only if (i) regarding explanatorily relevant properties, those properties of infinite limit systems disagree with the limiting properties of finite systems, (ii) infinite limit systems provide desired explanations, and (iii) the explanatory fuctions provided by the said properties of infinite limit systems are not equivalently provided by some other properties of finite systems.

Norton (2012) argues that when we have an infinite limit system and as we consider a property of the system, two situations arise: (a) the property of the infinite limit system agrees with the limiting property of finite systems, and (b) the property of the infinite limit system disagrees with the limiting property of finite systems. ${ }^{8}$ Norton (2012)'s examples of (a) and (b) are infinite sequences of capsules and ellipsoids where the property we consider is the ratio of surface area to volume (see Figures 1 and 2).

In case (a), we know that because the property of the infinite limit system and the limiting property of finite systems agree, the property of the limit system can be adequately expressed as the limiting property of finite systems. That is to say, statements about the property of this limit system can be expressed as approximations from the finite systems. Norton argues that in this case, the infinite limit system does not provide anything more than approximations of finite systems would offer. We can then safely conclude that in this case, infinite limit systems are dispensable (Norton, 2012, 211). ${ }^{9}$

\footnotetext{
${ }^{8}$ Norton (2012) also considers a third situation where there is no limit system, but since we are already in the context of infinite limit systems, I suppress that part of the discussion.

${ }^{9}$ One might worry that there are situations where a case (a) holds for some property, but the infinite limit system might still provide some "cognitive or epistemic goods" that are important or essential to the
} 


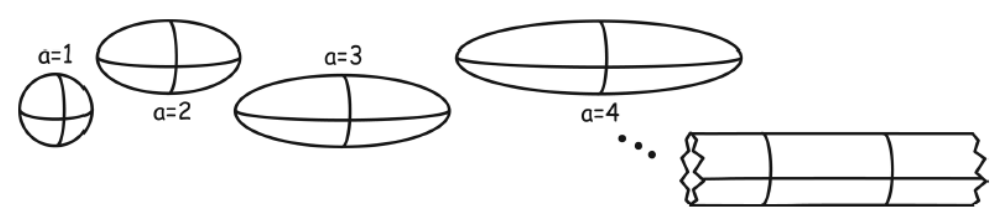

Figure 2: Unit sphere elongated into a sequence of ellipsoids. When the property we compare is the ratio of surface area to volume, the property of the limit system disagrees with the limiting property of sequence of capsules. (Norton, 2012)

In case (b), the property of the limit system and the limiting property of finite systems do not agree. From that Norton claims that the limit system is not a good candidate for idealization for the target systems because of its uncontrollable inexactitude (Norton, 2012, 215). ${ }^{10}$ For example, in some cases, infinite limit systems can exhibit "unphysical behaviors," blatantly violating physical laws such as determinism and conservation of energy (Norton, 2012, 215). It would be absurd for us to have "unphysical" infinite idealizations that would also provide proper inexact descriptions for our target systems.

While I agree with Norton's analysis in case (a) and recognize the problematic nature of infinite limit systems that exhibit unphysical properties in case (b), I nonetheless think that Norton's dismissal of infinite limit systems in case (b) is too quick. For one thing, not all infinite limit systems that fall in case (b) exhibit "unphysical behaviors." Some of the disagreements appear to be benign, or at least we should take them to be given the larger context. For example, all infinite limit systems trivially disagree with finite systems on one property: the (in)finitude of the parameter that we take to infinity to generate the infinite limit system. If we claim that infinite limit systems are unphysical qua being infinite, then it would be a strawman argument and we would lose the initial focus on infinite limit systems' role in the target scientific explanation. In fact, in RG explanations of universality, infinite limit systems do not exhibit outrageously unphysical behaviors on the scale of violating

explanation. I am fairly sympathetic to this worry, and I think in these situations, there would be some other property of infinite limit system that provides these "cognitive or epistemic goods," with respect to which a case (b) holds (i.e. the corresponding limiting property does not provide these goods). As I will suggest, in these situations, we would then want to examine whether this new property is explanatorily relevant, and whether these "cognitive or epistemic goods" can be equivalently provided by other properties of finite systems.

${ }^{10}$ See Sklar $(2000,61-71)$ for a discussion of "controllability" in the context of thermodynamics and limiting systems. Others have introduced similar distinctions as (un)controllability, such as "harmless and pernicious idealizations" in Earman (2008, 402) and "pathological/non-pathological idealizations" in Shech (2015). 
determinism or conservation of energy, even though they can be seen to fall in case (b) regarding the convergence property. In order to better understand systems in case (b) and their relationships to scientific explanation, we need a more fine-grained analysis.

I suggest that rather than asking whether the said property renders the entire system unphysical, we should ask whether the property of the infinite system is relevant to the explanation that the infinite system purports to offer. After all, we compare the properties between infinite and finite systems in order to decide whether infinite limit systems are dispensable in scientific explanations. On this understanding, the property in case (b) would render infinite limit systems indispensable only when it is central to the scientific explanation that infinite systems purport to offer. ${ }^{11}$

Furthermore, there is a special case of (b) worthy of attention, as it is reminiscent of case (a). This situation, which I call (b'), occurs when the property $\mathcal{P}$ of the infinite limit system and the limiting property $\mathcal{P}$ of finite systems do not agree. However, the property $\mathcal{P}$ serves an equivalent explanatory function in the target scientific explanation as another property $\mathcal{Q}$ of an finite system. In this case, I argue that infinite limit systems are also dispensable, because the explanatory functions that infinite limit systems offer can be recovered using finite systems.

In my view, explanatorily relevant properties provide certain explanatory functions in the explanation. It is moreover possible that multiple properties can provide the same explanatory functions in the explanation. Skipping ahead of ourselves a bit, in RG explanations of universality, the convergence property of infinite systems arguably provides explanatory functions by linking the original Hamiltonians to a Hamiltonian in the vicinity of a nontrivial fixed point, where we can recover empirically adequate values of critical exponents. What I will suggest in this paper is that we have reasons to believe that the linearization* property which I will define later also provides the same explanatory function under reasonable conditions. That is, the convergence property and the linearization* property can both effectively demarcate

\footnotetext{
${ }^{11}$ This is related to, but different from, Baron (2016)'s notion of "explanatory load-carrying" in the following way. When the statement "infinite limit systems have property $\mathcal{P}$ " carries explanatory load (i.e. had a system not have $\mathcal{P}$, then some features of the phenomana we want to explain would have been otherwise), then the property in case (b) is central to the scientific explanation in my sense. However, the converse is not true, as I explain in the next paragraph. $\mathcal{P}$ could be central to the explanation without the statement "infinite limit systems have $\mathcal{P}$ " carrying explanatory load. This is because another property of finite systems could provide the same explanatory function. I stick to my way of presentation since I want to highlight later in the paper the equivalent explanatory functions that different properties serve.
} 
universality classes. Showing that finite systems exhibit the linearization* property, we show a case (b') at work.

It follows that infinite limit systems are indispensable only when we have a case (b) regarding a property $\mathcal{P}$ such that $\mathcal{P}$ is central to the scientific explanation that infinite systems purport to offer and we do not have a case (b') regarding $\mathcal{P}$ and another property $\mathcal{Q}$. This clarifies what both sides of the debate needs to do. For an essentialist's view to be advanced, she needs to identify some explanatorily relevant property $\mathcal{P}$ for which case (b) holds and to show that $\mathcal{P}$ does not serve the same explanatory function as some other property $\mathcal{Q}$ of finite systems. On the other hand, for a dispensabilist's view to be advanced, she needs to identify all the explanatorily relevant properties and show that all of them fall into case (a) or (b').

For the rest of this paper, I will situate the debate on the role of infinite limit systems in RG explanations in the above framework. I will argue that an essentialist's position is not tenable on two grounds. First, the convergence property that they regard as crucial in RG explanations is less explanatorily relevant than the linearization* property of finite systems. Second, we have reasons to believe that when it comes to demarcating the universality classes, the explanatory functions served by convergence properties of infinite limit systems are equivalent to those served by linearization* property of finite systems, if certain conditions are met. This suggests that a case (b') is present. In the next section I will first consider the essentialists' argument while sketching the technical background of the RG method.

\section{RG Explanations: Argument for Indispensability?}

The essentialists maintain that in the case of RG explanations, behaviors of infinite limit systems cannot be retrieved by properties of finite systems (Batterman, 2017, 2019, 2005; Kadanoff, 2000). Kadanoff writes: "The existence of a phase transition requires an infinite system. No phase transitions occur in systems with a finite number of degrees of freedom" (Kadanoff, 2000, 238). Batterman argues that at the infinite limit, the behavior of the system is "qualitatively distinct" from the behavior of finite systems because the former is singular (Batterman, 2005, 235). Furthermore, Batterman claims in recent papers that the 
qualitatively different properties of infinite limit systems play necessary roles in explanations of our target phenomena-universality (Batterman, 2017, 2019). Batterman's view, if true, would suggest that infinite limit systems are indispensable precisely because some properties of the limit systems and the limiting properties of finite systems do not agree; these properties of the limit systems further our explanatory goals in ways that finite systems cannot.

In Sections 3.1 and 3.2, I will provide a technical background for the RG method to make salient the explanandum of RG explanations and discuss how infinite limits may play a role in the RG method. In Section 3.3, I will consider Batterman's defense of the necessity of infinite limit systems in RG explanations.

\subsection{Explanandum: Universality in Critical Phenomena}

Phase transitions are phenomenological changes in the bulk features of a physical system, often characterized by the discontinuities of some parameters of the system. Examples of phase transitions include the transformation from liquid to gas, or the transformation from a paramagnet to a ferromagnet. During a special type of phase transition-critical phase transition-some universal behavior "emerges" across microscopically different physical systems. Using the RG method, we can say why such universality occurs across these systems.

Consider the everyday phenomenon of water boiling. When water boils at room pressure, we usually observe air bubbles coming out. This phenomenon, called liquid-vapor coexistence, occurs due to the principal distinction between the densities of water and steam. However, when we vary pressure while keeping the water-steam system at its boiling temperature, at the critical point: a pressure of $22.064 M P a$ and temperature of $647 K$, the principal distinction between water and steam vanishes, and the boiling phenomenon disappears (Wilson, 1983, 103). Bubbles of steam and drops of water are intermixed at all length scales from macroscopic to atomic levels; the water-steam system then causes strong light scattering and displays a phenomenon called critical opalescence, where water and steam become milky (Wilson, 1983, 103).

The behaviors of the water-steam system near its critical point can be represented by the left side of Figure 3. Here, we use pressure and volume as the two variables, and supplement 

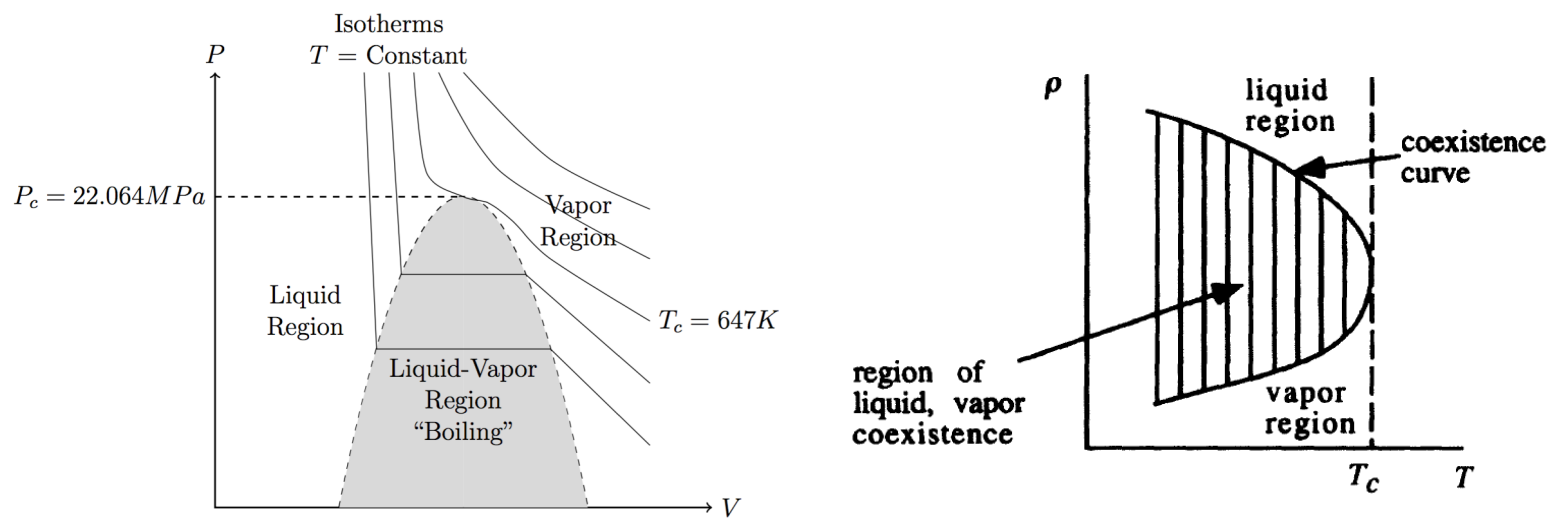

Figure 3: Left: $P V$ diagram with isothermal lines for the water-steam system near its critical point. $T_{c}$ represents the critical temperature, and $P_{c}$ represents the critical pressure. Right: The relationship between density difference and temperature. At $T<T_{c}$, the system within the coexistence curve is at the same pressure. (Batterman, 2000)

the graph with isotherms, i.e. points on the graph at the same temperature. At the critical point, the system undergoes a smooth phase transition; below the critical temperature, however, the system cannot undergo phase transitions without going through a region of liquid-vapor coexistence, where the temperature and pressure remain fixed, but the volume of the system expands. ${ }^{12}$

For the water-steam system at constant pressure and at boiling temperature below the critical point, volume difference can also be translated into density difference ${ }^{13}$ (See the right side of Figure 3). When the system approaches its critical point, the densities of water and steam converge. The curve that bounds the region of liquid-vapor coexistence is called the coexistence curve, and its shape can be represented by a power law relation between density difference and reduced temperature, the latter representing how close the system is to its critical temperature. We define the density difference $\Psi=\rho_{l i q}-\rho_{\text {vap }}$ and the reduced temperature $t=\frac{T-T_{c}}{T_{c}}$. We have the following power law relation

$$
\Psi \sim|t|^{\beta}
$$

for some $\beta$.

\footnotetext{
${ }^{12}$ Note that this is in the direction from liquid to vapor. Phenomenologically speaking, when the volume of the system expands, we observe air bubbles coming out.

${ }^{13}$ Here, we make the use of the fact that the overall mass of the system remains unchanged.
} 
Here $\beta$ is called a critical exponent of the water-steam system. The experimental value of $\beta$ is close to 0.33 . In fact, there is a set of critical exponents that describe the relationships between the reduced temperature and other physical properties. For example, the relationship between correlation length, the length scale at which local fluctuations in one part of the system affect those in another, and reduced temperature is given by

$$
\xi \sim|t|^{-v}
$$

A remarkable fact about critical phenomena is that regardless of the underlying microscopic structures, different fluids near their respective critical points essentially carry the same set of critical exponents. That is to say, the shapes of their respective coexistence curves are more or less the same. Furthermore, we know that physically more dissimilar systems also display identical critical phenomena categorized by the same set of critical exponents, if we make the appropriate change of parameters. The relationship between average magnetization and reduced temperature for an Ising model during its phase transition from a paramagnet to a ferromagnet is characterized by the same $\beta$ as that of water!

If critical behaviors of two physical systems are characterized by the same set of critical exponents, we say that they belong to the same universality class. Water, nickel, iron are all in the same universality class despite drastic differences in their microscopic compositions. Different physical systems are delimited into very few universality classes, which are hypothesized to be only based on properties like fundamental symmetries and dimensions of the order parameters (Kadanoff, 1971). ${ }^{14}$

Why do microscopically different physical systems within a universality class exhibit almost identical critical behaviors evidenced by their similar values of critical exponents? The RG method provides an account for this question. ${ }^{15}$ Accordingly, I regard the universal behaviors

\footnotetext{
${ }^{14}$ There is sometimes another related dependency question, namely why the categorization of universality classes only depends on fundamental symmetries and dimensions, that some scholars such as Batterman and Rice also take the RG method to explain (Batterman and Rice, 2014; Batterman, 2019). However, whether and how the RG method answers the dependency question is less clear. Those who claim that the RG method answers the dependency question nonetheless agree that the answer is a derivative, or a by-product, of how the universality class is delimited (Batterman and Rice, 2014, 363). I believe that to the extent that infinite limit systems are concerned, if an explanation of the stability question can be provided using finite systems, then an explanation of the dependency question can also be provided using finite systems.

${ }^{15}$ Note that this question is sometimes referred to by Batterman as a question about the autonomy of
} 
of systems within a universality class to be the explanandum of RG explanations. Successful explanans would tell a convincing story of how this universality happens. As we shall see, Batterman takes infinite limit systems to be an indispensable part of the explanans, a position that I will ultimately reject.

\subsection{The Use of Limits in the RG Method}

When we resort to the RG method to study critical phenomena of physical systems, we encounter two limits. The first limit is the thermodynamic limit as we take the number of particles to infinity. If a system is at its thermodynamic limit, we say that it is an infinite limit system. The second limit is the RG iterations limit as we take the number of RG iterations to infinity (Palacios, 2019, 619). The two limits are intricately connected. If we start with an infinite limit system, it converges to a nontrivial fixed point as we take the RG iterations limit. (Palacios, 2019, 619). ${ }^{16}$ Later in Section 3.3, I will present Batterman's argument that infinite limit systems are indispensable in RG explanations precisely because they have this convergence property that finite systems lack. Before presenting Batteman's argument, in the present section I will first introduce the RG method, and highlight the use of the two limits.

The thermodynamic limit comes into play in the RG method because, in order to deduce the discontinuities in the first or second derivatives of free energy, almost all successful statistical mechanical treatments of phase transitions require non-analycities in the free energy. However, the free energy of a system with finitely many particles is typically an analytic function. To represent systems undergoing phase transitions, we take the thermodynamic limit as the number of particles $N \rightarrow \infty$ to generate the needed non-analyticities. ${ }^{17}$

Near the critical phase transition, we work with systems with diverging correlation length, which, recall, measures how local fluctuations in one part of the system affect those in another. Since the relationship between the correlation length and the reduced temperature is given by macroscopic behaviors (Batterman, 2015, 2018).

${ }^{16}$ It is important to note that the two limits do not commute. See Palacios (2019) for further discussions.

${ }^{17}$ Note that the argument that phase transitions require the thermodynamics limit, though very widely accepted, is not a rigorous theorem; there are efforts to develop a theory of phase transitions for finite systems (see Butterfield (2011, 1124-25)). 
the following equation,

$$
\xi \sim|t|^{-v}
$$

where $v$ is typically positive, the correlation length diverges as $T \rightarrow T_{c}$, becoming effectively infinite at the critical point. The divergence of the correlation length as we approach the critical point of a system further implies a loss of characteristic length scale, and therefore local fluctuations at one part of the system are correlated with the entire system. The loss of characteristic length scale gives rise to the critical opalescence phenomenon in the water-steam system.

While the thermodynamic limit plays a role in the representation of systems near critical phase transitions, the RG iterations limit arises as we develop new methods to compute the parameters, including the critical exponents, of systems near criticality. Because of the long correlation lengths of systems near criticality, calculating the partition functions of individual systems is practically impossible in most cases due to the computational complexity. Furthermore, even if exact calculations of the partition functions are possible, we still have reasons to believe that simply showing that the critical exponents can be retrieved from microscopic functions is inadequate for our explanatory aspirations. After all, we would need an argument showing why there is a similar macroscopic behavior across systems, not an argument showing that systems individually do behave this way. In other words, we need an argument to connect the physical systems in a universality class with each other.

The RG method offers a viable alternative. In the RG method, we make use of the insights given by the scaling hypothesis, which claims that for systems near criticality, the correlation length is the only length scale that matters, and systems are scale invariant and self-similar (Simons, 1997). This allows us to apply RG transformations to convert a Hamiltonian that represents a system at a certain state to a Hamiltonian that is simpler but sufficiently similar to the original one. There are three steps in an RG transformation. We first coarse-grain the system by transforming the minimum length scale ${ }^{18} a$ to $b a$ where $b>1$, employing the fact that the system at a length scale $b a$ acts in some sense as a "single unit" as long as $a \ll b a \ll \xi$ (Goldenfeld, 1992). This step effectively reduces physical systems' microscopic

\footnotetext{
${ }^{18}$ For instance, the lattice spacing in an Ising model.
} 


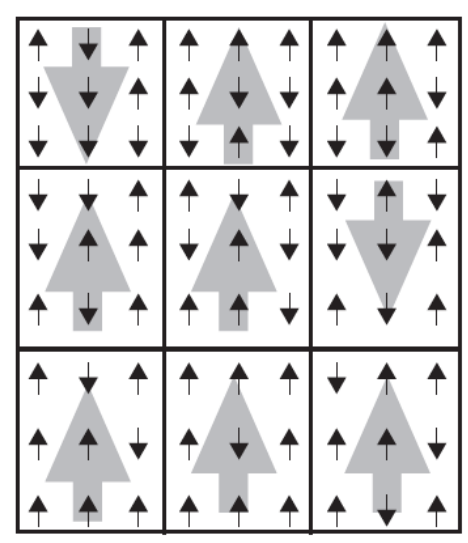

Figure 4: The coarse-graining step for the Ising model. (Kadanoff, 2010)

idiosyncrasies. Secondly, we rescale the model to restore the original "resolution." Finally, we renormalize the model to ensure that the Hamiltonian we retrieve after the RG transformation more or less describes the same physical system as before. The details of the three steps are the following:

- Coarse-grain: We multiply the minimum length scale $a$ of the system by a factor $b>1$. We do so by replacing the microscopic properties in the original area of size $(b a)^{d}$, where $d$ is the dimension of the physical system, with a coarser but representative microscopic property at the new minimum length scale $b a$, using some kind of "averaging" technique.

- Rescale: We restore the original resolution by dividing all length scales by a factor of $b$, because the new system is coarser than the original system.

- Renormalize: We adjust the variables ${ }^{19}$ in the new Hamiltonian such that the partition function and the free energy remain approximately invariant.

Figure 4 gives an example of an RG transformation. We have a two-dimensional Ising Model of a ferromagnet whose spins can either be up or down. We use a "majority rule" technique during the coarse-graining step, mandating that the new "block spin" of size $(b a)^{d}$ takes the direction of the majority of the original spins of size $a^{d}$. In this particular case, $b=3$ and $d=2$, so the new "block spin" takes the majority value of the 9 spins in its block. We then rescale the "block spins" to be reduced to the size of the original spins. The new picture would then be $\frac{1}{9}$ of its original size. We then compare other parameters of the new Hamiltonian with that of the old one, and renormalize the new Hamiltonian accordingly.

Next, we iterate the RG transformations to induce a sequence of Hamiltonians until we average out the entire correlation length. Let $S$ be the set of parameters that gives a com-

\footnotetext{
${ }^{19}$ For instance, the coupling constants in an Ising model.
} 


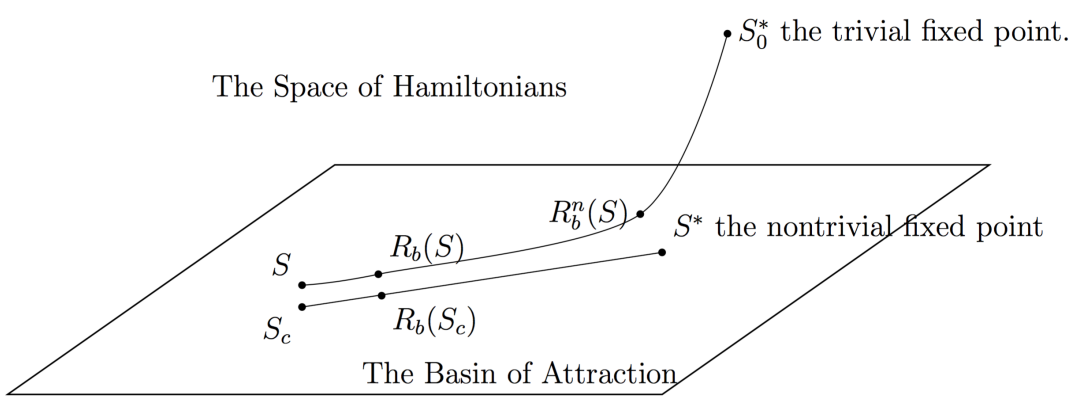

Figure 5: The RG trajectory of a finite Hamiltonian $S$ and an infinite Hamiltonian $S_{c}$. The RG trajectory of $S$ eventually converges to the trivial fixed point $S_{0}^{*}$. The RG trajectory of $S_{c}$ eventually converges to the nontrivial fixed point $S^{*}$.

plete description of a Hamiltonian. We denote by $R_{b}$ the RG transformation of factor $b$. The iterations of RG transformation can be represented by a RG trajectory in the space of Hamiltonians $^{20}$ (See Figure 5):

$$
S \rightarrow R_{b}(S) \rightarrow R_{b}^{2}(S) \rightarrow \cdots \rightarrow R_{b}^{n}(S) \rightarrow \cdots
$$

We study the critical behaviors by finding fixed points. Fixed points are Hamiltonians that stay intact after RG transformations, i.e. they are the Hamiltonians equipped with parameters $S^{*}$ such that $R_{b}\left(S^{*}\right)=S^{*}$. Recall that during the rescaling step, we divide all length scales of the system by a factor of $b$. We thus also divide the correlation length of a Hamiltonian by a factor of $b$ in a RG transformation. It follows that the fixed points either have a correlation length of $\xi\left(S^{*}\right)=\infty$ or $\xi\left(S^{*}\right)=0$, representing the high and low temperature fixed points respectively. The one fixed point with $\xi\left(S^{*}\right)=\infty$, called the nontrivial fixed point, is the one at criticality (the ones with $\xi\left(S^{*}\right)=0$ are called trivial fixed points).

Before preceeding further, let me add a comment regarding my notations. For the rest of the paper, I will use calligraphic letters such as $\mathcal{S}$ to represent physical systems, and regular letters such as $S, S^{\prime}$, or $S_{c}$ to represent an unrenormalized Hamiltonian of the system $\mathcal{S}$ when its parameters are at specific values, with $S_{c}$ representing the system $\mathcal{S}$ at its critical point. The two exceptions are $S^{*}$ and $S_{0}^{*}$, which represent the nontrivial fixed point and the trivial

\footnotetext{
${ }^{20} \mathrm{It}$ is important to note here that in the space of Hamiltonians, the unrenormalized Hamiltonians, i.e. the Hamiltonians that we start with, represent actual physical systems in an already idealized manner. The Hamiltonians on the RG trajectories are successively coarser representations of the physical systems.
} 
fixed points, respectively. Lastly, $R_{b}^{n}(S)$ represent the result of the $n$-th RG transformation with length scale $b$ of the point $S$. See Appendix $C$ for more details of my notations.

We can retrieve the critical exponents by a process I call linearization** ${ }^{21}$ Linearization* proceeds in the following, heuristic, way: we identify a point in the space of Hamiltonians, represented by $S^{*}+\delta S$, in the vicinity of the nontrivial fixed point; we then linearize on the RG transformation applied to this point. That is to say, we assume that the transformation $R_{b}\left(S^{*}+\delta S\right)$ is linear with regard to each parameter of $S$. We then use the semi-group properties $^{22}$ of the RG transformation to deduce eigenvectors and eigenvalues for $R_{b}$ regarding each parameter of $S$, and in turn correctly estimate the critical exponents. I explicate the details of the linearization* process in Appendix A.

Furthermore, each eigenvalue determines whether the respective eigenvector is a relevant eigenvector for the RG trajectories. The directions of RG trajectories away from the nontrivial fixed point are associated with the relevant eigenvectors, and the directions of RG trajectories towards the nontrivial fixed point are associated with the irrelevant eigenvectors (Goldenfeld, 1992, 246). The set of points that eventually flow into the nontrivial fixed points are called the basin of attraction. The RG transformation on these points only move in the irrelevant directions. On the other hand, the Hamiltonians that do not start in the basin of attraction would eventually flow away from it and converge to trivial fixed points (Palacios, 2019).

Two consequences of linearization* give us a basis for explaining universality in critical phenomena. By linearization*, we not only retrieve the critical exponents without working out the partition functions, but also define a basin of attraction on which physical systems after iterations of RG transformation flow to the nontrivial fixed point. Each nontrivial fixed point thus delimits a universality class by the set of critical exponents that one obtains from linearizing* around it. The above facts allow us to claim that different physical systems fall in the same universality class and exhibit universal behaviors characterized by their critical

\footnotetext{
${ }^{21}$ The asterisk is introduced to distinguish linearization* from ordinary linearization of the RG transformation. Linearization* specifically refers to linearization in the vicinity of the nontrivial fixed point such that we can retrieve reliable critical exponents.

${ }^{22}$ We regard each RG transformation, $R_{b}$, as an element of a semi-group. By definition of a semi-group, there is an associative group operation on elements: $R_{b b^{\prime}}=R_{b} R_{b^{\prime}}$. Each element does not necessarily have an inverse. The semi-group property of RG transformations explains (in a folk usage of the word) the name "group" in Renormalization Group.
} 


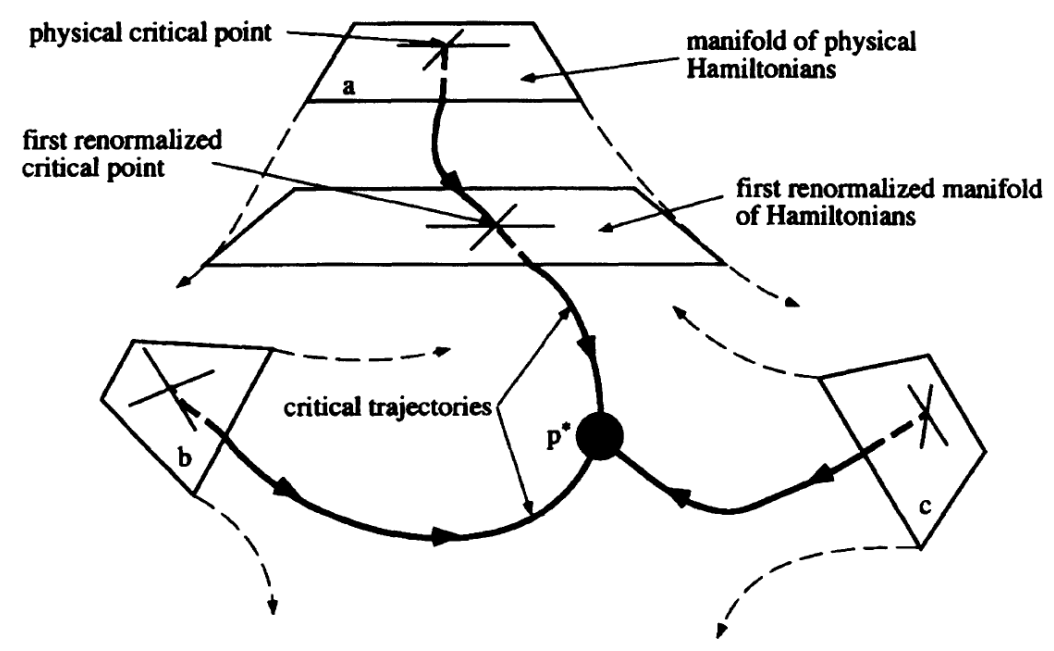

Figure 6: The RG trajectories of Hamiltonians representing different systems flowing towards the nontrivial fixed point $p *$. These Hamiltonians are all in the basin of attraction. (Batterman, 2000)

exponents, because their RG trajectories flow towards the same nontrivial fixed point in the basin of attraction (See Figure 6).

Importantly, because the correlation length at the nontrivial fixed point is infinite, the systems in the basin of attraction also have infinite correlation length. This is again because the correlation length $\xi$ is divided by a factor of $b$ during each RG transformation. ${ }^{23}$ Only for systems with infinite correlation length does the RG transformation not reduce their correlation length, i.e. $\xi\left(R_{b}(S)\right)=\xi(S)=\infty$. The systems in the basin of attraction are at their critical points. To represent these systems, we need to take the thermodynamic limit.

Averaging out an infinite correlation length requires infinite iterations of RG transformations. Taking the number of iterations of RG transformations to infinity, the physical systems in the basin of attraction converge to the nontrivial fixed point. This is the second limit we utilize in the RG process: the infinite iterations of RG transformations.

\footnotetext{
${ }^{23}$ This also explains why the Hamiltonians starting off the basin of attraction move further and further away from the basin of attraction. The relation $\xi \sim|t|^{-v}$ entails that the original systems with finite correlation length moves further and further away from the critical point.
} 


\subsection{Indispensability of Infinite Limit Systems? The Convergence Property}

Batterman argues that the limit system is indispensable precisely because a property of infinite systems and the limiting property of finite systems do not agree. For him, this property of infinite systems plays relevant roles in explanations of universality.

The property that Batterman has in mind is whether the systems converge to the nontrivial fixed point after infinite iterations of RG transformations. He writes,

[c]rucially, those systems that actually flow to a fixed point are at criticality. This means that they are infinite systems. As such, those systems are idealizations. But the infinite idealization is necessary if one is to locate the fixed points of the $\mathrm{RG}$ flow in the abstract space. This is because the correlation length must diverge to be able to infinitely iterate the RG transformation. (Batterman, 2019, 39)

I say that a system's convergence property is "convergent" if the system eventually converges to the nontrivial fixed point, and "non-convergent" otherwise. In the RG methods, infinite limit systems eventually converge to the nontrivial fixed point after taking the RG iterations limit. But finite systems, no matter how large, do not converge to the nontrivial fixed point. If the convergence property is what we are concerned with, the limiting property of finite systems returns "non-convergent," while that of the infinite limit system is "convergent." Here, the property of the infinite limit system and the limiting property disagree. The convergence property gives rise to case (b) as discussed in Section 2.

Batterman $(2017,571)$ then argues that "explanations of the behavior of real finite systems requires the use of mathematical infinities." Continuing in the footnote, he states "if we want to explain the universal behavior of large but finite systems using the RG, then we need to find a fixed point and, to my knowledge, this requires an infinite system" (Batterman, 2017, $571)$.

Batterman's defense of the indispensability of the infinite limit systems really comes down to arguing for the explanatory relevance of the convergence property in RG explanations. Finding the fixed point, he claims, is necessary in explanations of large but finite systems, and could only be done using infinite systems. However, as I will argue in the next section, finding 
the fixed point can be seen as neither necessary nor sufficient in explaining universality. What is important in explanations of universality is a story about how different systems come to behave in similar ways, a story the linearization* property, as I define presently, can effectively tell.

\section{The Linearization* Property}

In this section, I will first make a few arguments against the explanatory relevance of the convergence property. These arguments, in turn, lead me to define and adopt the linearization* property, which as I argue is more explanatorily relevant than the convergence property. After defining the linearization* property, I will explicitly define what it means for a physical system to be in a universality class using the linearization* property. At the end of this section, I will state a conjecture on the equivalence of the convergence property and the linearization* property with respect to the explanatory function of demarcating the universality classes. In the next section, I will present both heuristic and technical evidence inspired by Wilson and Kogut (1974) and Yin (2011) to motivate and prove a proposition demonstrating that, under reasonable conditions, the conjecture holds.

A crucial consequence of my analysis is that, in my view, only Hamiltonians off the basin of attraction exhibit the linearization* property. As a result, my conjecture implies the possibility of a case ( $\left.b^{\prime}\right)$ as discussed in Section 2, where an explanatory functiondemarcating the universality classes-as provided by convergence properties of infinite limit systems is equivalent to those provided by linearization* properties of finite systems. When the latter property is more explanatorily relevant for other reasons, we have a strong case to deem the former property dispensable in RG explanations. A fortiori, infinite limit systems are merely approximations, and therefore dispensable.

For the essentialists, the convergence property is explanatorily relevant because points with the convergence property flows to the nontrivial fixed point. But why is the nontrivial fixed point relevant for the explanation? Batterman (2000) suggests that this is due to the linearization* process. He writes 
One can perform an analysis (linearization) in the neighborhood of such a fixed point which shows how the renormalization transformation acts on points which differ only slightly from the fixed point itself. This yields local axes which determine how lengths are scaled in different directions around the fixed point. In turn, this analysis allows for the calculation of the critical exponents. The fixed point describes, therefore, a specific critical behavior whose salient properties are determined by the local behavior of the renormalization group transformation around the fixed point. Furthermore, and this is where we finally get an understanding of the universality of this critical behavior, corresponding to each fixed point is a domain of attraction. These are all those points in the abstract parameter space (the space of all Hamiltonians) which, under transformation... eventually flow into the fixed point. (Batterman, 2000, 142, emphasis in original)

Morrison too, describes the importance of the nontrivial fixed point by appealing to the linearization* process

The calculation of values for critical indices ... is the foundation of universality. RG is the only means possible for explaning that behaviour... [L]inearization around a fixed point will certainly tell you about finite systems, but the fixed point itself requires the limit. (Morrison, 2015, 100)

Batterman and Morrison's comments above align with my discussions of the linearization* process in Section 3.2 and in more detail in Appendix A. The linearization* process gives us information about the critical exponents and the basin of attraction. ${ }^{24}$ This forms a basis for RG explanations because the value of the critical exponents that we calculate from the linearization* process matches our experimental result. For essentialists like Batterman and Morrison, the linearization* process support the explanatory relevance of the nontrivial fixed point and thus the convergence property in the following way: critical points in the basin of attraction converge to the nontrivial fixed point; moreover, the nontrivial fixed points are relevant in RG explanations because linearizing* around it gives us the critical exponents that demarcate universality classes. For them, the indispensability of the infinite limit systems comes as a consequence of the above analysis-the Hamiltonians representing the critical points are necessarily infinite, and therefore infinite limit systems are indispensable.

However, I think the essentialists' reasoning is not entirely satisfactory. For one thing, if linearizing* around the nontrivial fixed point is doing all the work for retrieving the critical exponents and demarcating the universal classes, then what is so special about converging to

\footnotetext{
${ }^{24}$ Domain of attraction mentioned in the Batterman quote above is equivalent to the basin of attraction.
} 
the nontrivial fixed point itself? Granted that mathematically speaking, the closer the point at which we linearize* is to the nontrivial fixed point, the more accurate the calculation of critical exponents would be, but it is simply impossible to linearize* at a fixed point, since by definition, fixed points remain fixed after RG transformations. ${ }^{25}$ This suggests that if we want to take the linearization* process seriously, we should take the neighborhood around the nontrivial fixed point seriously, rather than the nontrivial fixed point itself.

This brings up a further point about a gap that needs to be filled in the reasoning adopted by the essentialists. For essentialists like Batterman, a Hamiltonian at its critical point is crucial to the RG explanations. However, this Hamiltonian is only one, albeit important, Hamiltonian that constitutes the system's behaviors near criticality. It does not give us a full picture of the critical phenomena. In order to obtain the critical exponents both experimentally and through the RG method, we need data from other Hamiltonians near, but not at the critical points. For the essentialist's position to be tenable, they need additional arguments about how the particular behaviors of the Hamiltonian at the critical point generalize to the Hamiltonians near criticality, i.e. how the convergence of the Hamiltonians in the basin of attraction tells us a story about systems in a universality class, whose behaviors can only be fully characterized if we also have data from Hamiltonians close to, but not at the critical points. I think this challenge is a hard one to tackle for the essentialists because it generates a dilemma-if the convergence property is really so crucial to the explanation, then how are the behaviors of the Hamiltonians that converge to the nontrivial fixed point supposed to generalize to the behaviors of the Hamiltonians that do not? If, on the other hand, we can learn something about the Hamiltonians close to criticality from the Hamiltonians at their critical points, then is it really the convergence, or is it something else, that allows for this generalization? I'd like to suggest that these are serious concerns that need to be adequately addressed. Building off my previous discussions, my response to the dilemma is to suggest that it is linearization*, rather than convergence, that is doing the explanatory work here.

Furthermore, let me add a technical observation about the linearization* process. In an

\footnotetext{
${ }^{25}$ Of course we can linearize at the nontrivial fixed point, the result will just be trivial. What I claim is the impossibility to perform linearization*, with an asterisk, at the point. Linearization* entails that the results should give us meaningful information about the critical exponents of the universality class that the fixed point delimits. In this sense, linearization* on the RG transformation of a fixed point is impossible.
} 
important sense, the linearization* process privileges Hamiltonians off the basin of attraction as compared to Hamiltonians in the basin of attraction. To see why, recall that in the linearization* process (see Appendix A for details), we start with a point $S^{*}+\delta S$ in the vicinity of the nontrivial fixed point, linearize on the RG transformation at that point, and then demarcate eigenvectors of the RG transformation matrix as relevant, irrelevant, and marginal eigenvectors based on their respective eigenvalues. If the point that we linearize at is in the basin of attraction, then it follows that $\delta S_{i}=0$ if $O_{i}$ is a relevant or marginal eigenvector. Linearizing at such a point would not give us information about the directions other than those in the basin of attraction. This fact becomes salient when we calculate the critical exponents. This is because the reduced temperature, which is the base of the power relations for multiple parameters, is in the direction of a relevant eigenvector. In order to retrieve the critical exponents through the linearization* process, we have to consider points that have nonzero reduced temperature. These points cannot be infinite limit systems in the basin of attraction.

I have so far argued that, first, since the linearization* process and the calculation of critical exponents are crucial in the explanation, as the essentialists seem to agree, then we should focus on the neighborhood around the nontrivial fixed point where linearization* is possible rather than the fixed point itself; furthermore, we can only calculate the critical exponents by linearizing* at the points off of the basin of attraction. Since points with the convergence property are all in the basin of attraction, the convergence property is not entirely relevant in this crucial part of the explanation. Can we define a more explanatorily relevant property that makes use of the linearization* process, such that finite systems off of the basin of attraction could satisfy it? I suggest that we can. To define such a linearization* property, however, there are several hurdles to clear.

First, in order to define a linearization* property for Hamiltonians that represent actual physical systems, we need to specify how these Hamiltonians in the vicinity of the nontrivial fixed point connect back to Hamiltonians that represent actual physical systems-the unrenormalized ones-close to their critical points. I suggest that, like how a Hamiltonian's convergence property is determined, the connection can come from some "interactions" of 
the RG trajectories of unrenormalized Hamiltonians and a neighborhood around the nontrivial fixed point where the linearization* process is possible. I will call this neighborhood the linearization*-adequate neighborhood for the rest of this paper. It is worth noting that exactly what the linearization*-adequate neighborhood looks like is an empirical question that we currently lack the answer to, though we know that the neighborhood would contain points off the basin of attraction that are still "very close" to the nontrivial fixed point. What points would fall within the neighborhood depends partly on our tolerance of imprecision as well, i.e. how closely the retrieved values of critical exponents through linearizing on these points should match the experimentally measured values of critical exponents. Later, after I specify the mathematical structures of the space of Hamiltonians, I will put forward reasonable conditions about the topological properties of the linearization*-adequate neighborhood, which then allows us to prove deductive consequences related to my conjecture, which I will also introduce shortly.

Palacios (2019) also draws attention to the importance of the neighborhood around the nontrivial fixed point in the RG process, but she does not further specify how this neighborhood "interacts" with the RG trajectories. Indeed, a challenge arises when we press on the details of the aforementioned "interactions." One possibility would be to require that a Hamiltonian exhibits the linearization* property if its RG trajectory converges to some point in the linearization*-adequate neighborhood. However, this requirement is too strong because it makes the set of Hamiltonians that satisfy the linearization* property empty. To see why, recall that the Hamiltonians that start off the basin of attraction would eventually flow away from the nontrivial fixed point towards a trivial fixed point, so these Hamiltonians would eventually leave the linearization*-adequate neighborhood. The infinite limit systems that start in the basin of attraction, however, would not flow into a linearization*-adequate neighborhood for reasons discussed before-points in the basin of attraction lack components in the direction of the reduced temperature, and are therefore inadequate to be used to retrieve the critical exponents through the linearization* process. Alternatively, we can require that a Hamiltonian exhibits the linearization* property if its RG trajectory intersects with the linearization*-adequate neighborhood. However, one might worry that this requirement is 


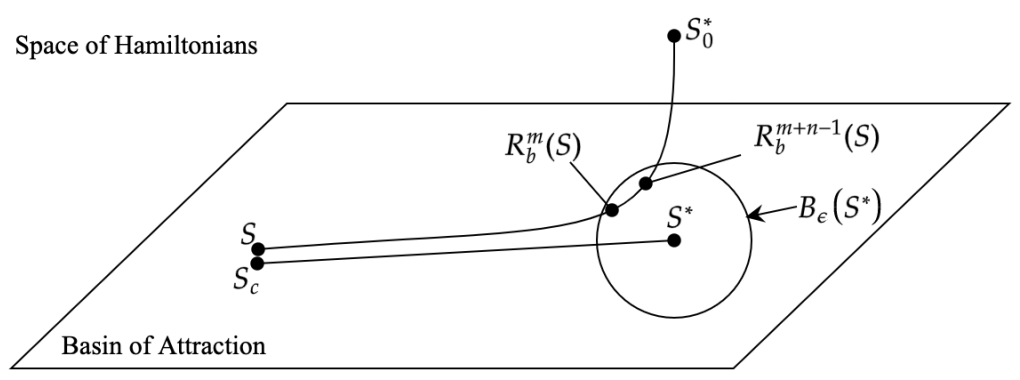

Figure 7: $S$ exhibits the linearization* property.

too permissive. For example, what if the RG trajectory of a Hamiltonian passes through a linearization*-adequate neighborhood of a nontrivial fixed point just by accident, and it would stay longer at a linearization*-adequate neighborhood of another nontrivial fixed point? ${ }^{26}$ To avoid the potential drawbacks of the two extremes, I suggest that we adopt the following definition of the linearization* property:

Definition 1 (Linearization* Property). Given a linearization*-adequate neighborhood $B_{\epsilon}\left(S^{*}\right)$ of $S^{*}$ and an arbitrary natural number $n$, a Hamiltonian $S$ exhibits the linearization* property with respect to $B_{\epsilon}\left(S^{*}\right)$ and $n$ iff there exists $m \in \mathbb{N}$ such that $R_{b}^{m+i}(S) \in B_{\epsilon}\left(S^{*}\right)$ for all $0 \leq i<n$ and $i \in \mathbb{N}$.

This definition, together with a later definition of Universality Class that quantifies over $n$, captures the sense in which the RG trajectory of a Hamiltonian stays "as long as one likes"as measured by the number of RG iterations-in the vicinity of the nontrivial fixed point, so that it is not an accident that the RG trajectory passes through the linearization*-adequate neighborhood (see Figure 7 for an illustration of a Hamiltonian exhibiting the linearization* property).

Having defined the linearization* property, I now turn to the task of explanatorily connecting the linearization* property to our explanandum-the universality question. Specifically, my goal is to spell out conditions for a system to be in a particular universality class using

\footnotetext{
${ }^{26}$ To be fair, I have not encountered any discussions in the literature suggesting that this situation is in fact possible, and it might well be the case that this requirement would turn out to be equivalent to the prima facie stronger requirement that I define next. However, I still consider this possibility to preemptively rule out potential counterexamples.
} 
the linearization* property. To accomplish this task, we also need to make sure that we fill the gap in the essentialists' RG explanations that I identified earlier. Namely, instead of only talking about the behaviors of critical points, we ought to make explicit reference to the Hamiltonians that are very close to being critical as well, as they crucially contribute to the critical phenomena and play an important role in the experimental measurements of critical exponents.

To formally express notions of closeness and convergence, some specifications about the mathematical structures of the space of Hamiltonians are in order. First, I require that the space at each step of the RG transformation is a Banach space; ${ }^{27}$ that is, it is a complete space equipped with a norm, though the norms at different steps of the RG transformation may differ. I use $R_{b}^{n}$ to denote the surface at the $n$-th step of the RG transformation with length $b$ and I use $\|\cdot\|_{b}^{n}$ to denote the norm on the $R_{b}^{n}$ surface. Moreover, I use $\|\cdot\|$ to denote the norm on the unrenormalized surface. The norms induce topologies on the surfaces. For example, we can define an open neighborhood $N\left(R_{b}^{n}(S), r\right)$ around $R_{b}^{n}(S)$ on the $R_{b}^{n}$ surface as the set of points $Q$ on the surface such that $\left\|Q-R_{b}^{n}(S)\right\|_{b}^{n}<r$. Secondly, I require that the basin of attraction is equipped with a topology $\mathcal{T}$. For every point $S$ on the unrenormalized surface, $R_{b}^{n}(S)$ is in the $R_{b}^{n}$ surface, but we do not make the requirement in the opposite direction-it doesn't have to be the case that for every point $P$ in the $R_{b}^{n}$ surface, there is an $S$ on the unrenormalized surface such that $R_{b}^{n}(S)=P$. Figure 8 is an illustration of the mathematical structures of the space of Hamiltonians. Note that under this specification, there is not a formal way to measure the distance between points in the space of Hamiltonians that belong to two different steps of RG transformations.

It is important to note here that the above specifications of the mathematical structures are not standardly discussed in the literature, in the following sense: although some authors (e.g. Yin (2011)) introduce norms on the $R_{b}^{n}$ surfaces, there are very few discussions, let alone agreements, on which norms are appropriate to use. Moreover, numerous authors discuss the convergence of $R_{b}^{n}\left(S_{c}\right)$ to $S^{*}$ as $n \rightarrow \infty$ in the basin of attraction. But, they have not specified the mathematical space in the basin of attraction in which the convergence is defined. By

\footnotetext{
${ }^{27}$ As an example of this, Yin (2011) constructs a Banach space from the interaction terms of an Ising model.
} 


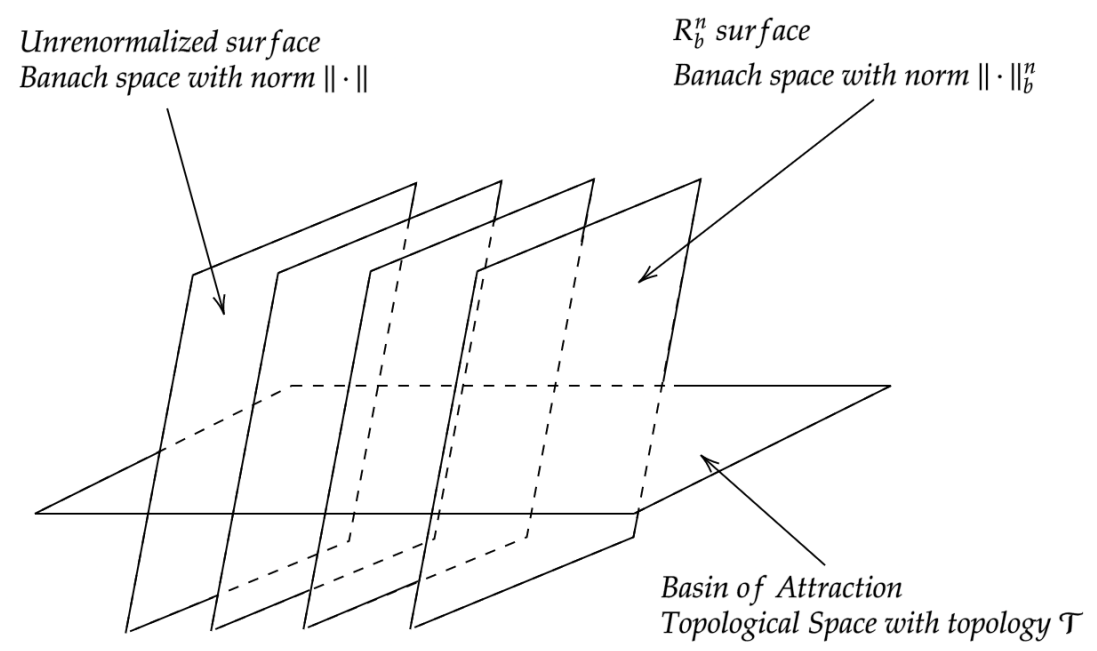

Figure 8: The mathematical Structures of the space of Hamiltonians for one system.

introducing mathematical structures on the space of Hamiltonians, I merely hope to stipulate some underlying structures in order to (1) make sense of the technical claims and conjectures that various authors have made about the space of Hamiltonians, and to (2) formulate and prove statements about the linearization* property. By saying that there are norms on the $R_{b}^{n}$ surfaces, and there is a topology in the basin of attraction, I remain agnostic about exactly what these norms and the topology are. ${ }^{28}$ In my opinion, we are in need of a separate but important philosophical discussion about which norms and which topology are appropriate to use for the $R_{b}^{n}$ surfaces and the basin of attraction, respectively. However, in the absence of such a discussion, I proceed with the supposition that there are norms on the $R_{b}^{n}$ surfaces to make sense of how close points are to each other, and there is a topology in the basin of attraction to make sense of convergence. None of my definitions, propositions, and conjecture is dependent on particular choices of norms and topologies.

I will now define what it means for a system to be in a particular universality class delimited by a nontrivial fixed point.

Definition 2 (Universality Class). A physical system $\mathcal{S}$ is in the universality class delimited by a nontrivial fixed point $S^{*}$ iff for any linearization*-adequate neighborhood $B_{\epsilon}\left(S^{*}\right)$ of $S^{*}$, there exists $m \in \mathbb{N}$ such that, for any arbitrary natural number $n$, there is a Hamiltonian

\footnotetext{
${ }^{28}$ Or whether we can do away with the norms and introduce a metric on the space.
} 
$S$ representing $\mathcal{S}$ with $S \notin B o A$ such that for all Hamiltonians $S^{\prime}$ representing $\mathcal{S}$ with $0<\left\|S^{\prime}-S_{c}\right\| \leq\left\|S-S_{c}\right\|$ and $S^{\prime} \notin B o A$, we have $R_{b}^{m+i}\left(S^{\prime}\right) \in B_{\epsilon}\left(S^{*}\right)$ for all $0 \leq i<n$ and $i \in \mathbb{N}$.

In this definition and hereafter, $B o A$ stands for the basin of attraction. Moreover, we can think of the systems that are closer to the critical points as those whose reduced temperature is getting smaller, i.e. they are closer and closer to the critical temperature. Given this definition, the following Proposition immediately follows.

Proposition 1. If a system $\mathcal{S}$ is in the universality class delimited by a nontrivial fixed point $S^{*}$, then for any linearization*-adequate neighborhood $B_{\epsilon}\left(S^{*}\right)$ of $S^{*}$ and any arbitrary natural number $n$, there exists a Hamiltonian $S$ representing $\mathcal{S}$ with $S \notin B o A$ such that all Hamiltonians $S^{\prime}$ representing $\mathcal{S}$ with $0<\left\|S^{\prime}-S_{c}\right\| \leq\left\|S-S_{c}\right\|$ and $S^{\prime} \notin B o A$ exhibits the linearization* property with respect to $B_{\epsilon}\left(S^{*}\right)$ and $n$.

Proof. See Appendix B.

It follows from Propsition 1 that if a physical system is in a particular universality class, then there is a set of Hamiltonians very close to the critical point that exhibit the linearization* property. These points are crucial in mapping out the coexistance curve powered by the critical exponents experimentally. Correspondingly, under my definition, the RG trajectories of these points are crucial in calculating the critical exponents through the linearization* process. I believe that this correspondence signals an adequate explanatory connection between the linearization* property and the explanandum.

Lastly, we need to show that Definition 2 correctly demarcates the universality classes. By that I mean that physical systems that belong to a universality class under my definition are the same as the ones that belong to the said class in the received approach. According to the received approach, physical systems are in the same universality class if points at their critical points converge to the same nontrivial fixed point after iterations of RG transformations. This leads to the following conjecture.

Conjecture 1. Given a physical system $\mathcal{S}, S_{c}$ representing $\mathcal{S}$ at its critical point, and a nontrivial fixed point $S^{*}, R_{b}^{n}\left(S_{c}\right) \rightarrow S^{*}$ as $n \rightarrow \infty$ iff $\mathcal{S}$ is in the universality class delimited by $S^{*}$ under Definition 2. 
The consequence of this conjecture is as follows. We know from the discussions leading up to Definition 1 that the Hamiltonians that could possibly exhibit the linearization* property are finite systems, though possibly large, close to the critical points. Therefore, if Conjecture 1 is true, then the explanatory function in demarcating universality classes that infinite limit systems would deliver via the convergence property is fully equivalent to what large but finite systems would provide through the linearization* property. This would lead us to a case (b') as discussed in Section 2. Furthermore, I have argued throughout the section that delimiting universality classes according to the linearization* property offers a stronger explanatory connection to the explanandum than the convergence property, because the former accounts for the behaviors of a whole set of points close to being critical, which are crucial in describing the critical phenomena. This suggests that the linearization* property is more explanatorily relevant than the convergence property. If Conjecture 1 is true, we would have ample reasons to think that not only infinite limit systems are dispensable in the RG explanations, large but finite systems actually offer more adequate and relevant explanations.

\section{The Dispensability of Infinite Limit Systems}

In this section, I will first present heuristic evidence from Wilson and Kogut (1974) suggesting the plausibility of Conjecture 1. I will then present technical evidnce from Yin (2011) to motivate a key condition for the conjecture. Furthermore, I will introduce a few reasonable auxiliary conditions about the space of Hamiltonians and the linearization*-adequate neighborhood. Finally, I will prove that these conditions are sufficient for Conjecture 1.

Wilson and Kogut $(1974,164)$, pioneers in developing the RG method, suggest that for a large but finite system infinitesimally close to the critical point, its RG trajectory will flow extremely close to the basin of attraction almost to the nontrivial fixed point, but eventually drives itself away to converge to a trivial fixed point. They show this by providing an analogous classical mechanics case in which a ball rolls down a hill (See Figure 9 ). Here $P_{\infty}$ represents a saddle point, and $P_{0}$ represents the absolute minimum point. These two points are analogous to the nontrivial fixed point and the trivial fixed point, respectively. Moreover, $R$ represents 
the ridge line, which is analogous to the basin of attraction in the space of Hamiltonians. In this case, if we roll a ball on the ridge line $R$, it rolls down to the saddle point $P_{\infty}$. However, if we roll a ball to the west of the ridge line $R$, the ball rolls to the bottom of the hill $P_{0}$. An interesting phenomenon occurs if we place the ball infinitesimally close to, but to the west of, the ridge line $R$. It rolls down close to the ridge line almost to the saddle point. Near the saddle point the ball rolls very slowly because the terrain is almost flat. But eventually the ball veers away from the ridge line and rolls down to $P_{0}$ (Wilson and Kogut, 1974, 164-165). For Wilson and Kogut (1974), this is analogous to a large but finite system starting infinitesimally close to the critical point. If this analogy is correct, then the RG trajectory of this finite system would first flow very close to the basin of attraction almost to the nontrivial fixed point, stay there for numerous iterations of RG transformations, until it eventually turns and flows to a trivial fixed point. For finite points even closer to the basin of attraction, we would expect to see them flow much closer to the basin of attraction and the nontrivial fixed point and stay for an even larger number of RG iterations, until they rapidly drive away to the trivial fixed point. This phenomenon about the different behaviors of a point very close to the critical surface and a point much farther away is now referred to as crossover (see Mainwood (2006, Section 4.4.2) and Butterfield (2011, Section 7.2.2) for philosophical discussions of crossover, and Cardy (1996, 69-71) for technical details). ${ }^{29}$

Wilson and Kogut (1974)'s work provides heuristic evidence for Conjecture 1. In the classical mechanics case, if we pick an arbitrary neighborhood containing the saddle point $P_{\infty}$, we know that we can put a ball at a position very close to the ridge line such that it will roll into the neighborhood and stay there for a long time before it rolls away from the neighborhood. If we put the ball even closer to the ridge line, the ball will roll into the neighborhood and stay for a longer time until it begins to roll down. If the analogy applies,

\footnotetext{
${ }^{29}$ Mainwood (2006) and Butterfield (2011) have previously used finite-size crossover to support dispensabilism. Finite-size crossover describes a situation where, for a system of finite size, if its correlation length is small compared to the size of the system, but very large compared to minimum length scale, the system flows towards a fixed point associated with the infinite system. However, when the correlation length is comparable to the size of the finite system, then the flow crosses over to some other fixed point (Mainwood, 2006, 244; Butterfield 2011, 1131-32). This phenomenon lends support to my main argument. As the correlation length grows large, i.e. as we get closer to the critical phase transition, as long as we have a finite system whose size is still large compared to the correlation length, finite-size crossover tells us that its RG trajectory will flow towards the (right) fixed point (until it is driven away).
} 


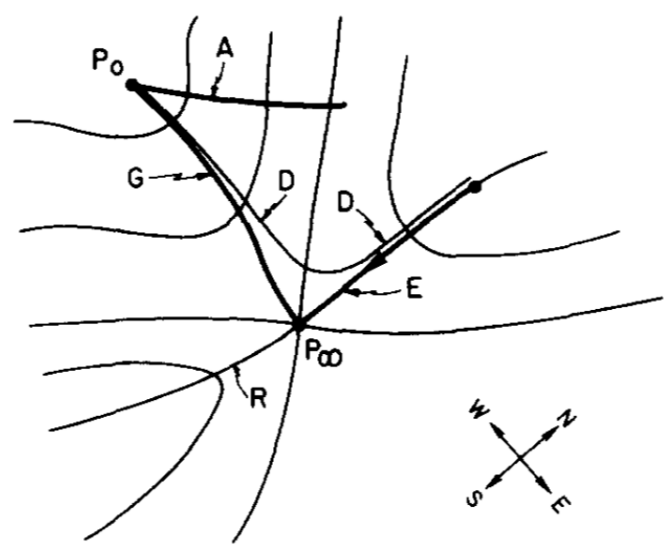

Figure 9: A ball rolls down the hill. $P_{\infty}$ represents the saddle point, $P_{0}$ represents the absolute minimum, and $R$ represents the ridge line. (Wilson and Kogut, 1974)

then in the RG case, for every arbitrary linearization*-adequate neighborhood, we should be able to find a Hamiltonian very close to being critical such that its RG trajectory flows into the linearization*-adequate neighborhood for a large number of iterations before leaving. Moreover, the RG trajectories of the Hamiltonians closer to the basin of attraction would also enter the neighborhood and stay for even longer as measured by the number of RG iterations. In this case, the target system would be in the universality class delimited by the nontrivial fixed point according to Definition 2. We also know that here Hamiltonians at their critical points flow into the nontrivial fixed point because of the saddle topography in the analogous case. Therefore, our system is also in the same universality class according to the traditional definition. It follows that Conjecture 1 is plausible as long as Wilson and Kogut (1974)'s analogy is applicable.

Yin (2011)'s recent work provides relevant formal support for Conjecture 1. She agrees with Wilson and Kogut's characterizations of the qualitative behaviors of finite systems starting infinitesimally close to the critical point. She writes,

[f]or a point not on the critical surface but very close to being critical, the RG map will first drive it towards the fixed point for a large number of iterations, but eventually will drive it away. (Yin, 2011, 1) 30 $^{30}$

Yin then builds upon the results by Haller and Kennedy (1996) in formalizing the mathematical structures on the space of Hamiltonians for an Ising model, providing a norm at each step

\footnotetext{
${ }^{30}$ See also Cardy $(1996,69)$ and Mainwood $(2006,166)$ for similar claims.
} 
of the RG transformation. Under a hypothesis regarding high-temperature phase uniformity, which is tested to be true in representative special cases such as the decimation transformation and the Kadanoff transformation, Yin is able to derive an upper bound for the linearization of each RG transformation (Yin, 2011, 3,11). Here, linearization should be distinguished from linearization*. The former does not imply that we can retrieve reliable critical exponents from it.

To elaborate on Yin's result, she claims that for a Hamiltonian infinitesimally close to the critical point, ${ }^{31}$ represented by $S=S_{c}+K$, where $K$ is the deviation from this critical point, its behavior under RG transformation can be governed by a linearization

$$
R_{b}(S)=R_{b}\left(S_{c}+K\right)=R_{b}\left(S_{c}\right)+L+\text { corrections. }
$$

Here, $L$ is the linearization on the RG transformation from $S$ to $R_{b}(S)$, which, roughly speaking, measures the distance between $R_{b}(S)$ and the renormalized critical point (Yin, 2011, 3). Given the above hypothesis, Yin was able to prove that $L$ has an upper bound which can be made small (Yin, 2011, 11).

Though Yin (2011)'s work is set in a simple Ising model case, her results suggest that a generalized condition, stated below, is plausible for all physical systems near critical phase transitions. This condition serves as a key condition for proving Conjecture 1.

Condition 1. For all $r \in \mathbb{R}^{+}, b>1$, and $S_{c}$ representing physical system $\mathcal{S}$ at its critical point, there exists $S$ representing $\mathcal{S}$ with $S \notin B o A$ such that $0<\left\|R_{b}(S)-R_{b}\left(S_{c}\right)\right\|<r{ }^{32}$

I now introduce a few more reasonable auxiliary conditions on the space of Hamiltonians and the linearization*-adequate neighborhood.

Condition 2. For all $S, S^{\prime} \notin B o A$ representing physical system $\mathcal{S}$ and $S_{c}$ representing $\mathcal{S}$ at its critical point, if $0<\left\|S^{\prime}-S_{c}\right\| \leq\left\|S-S_{c}\right\|$, then $0<\left\|R_{b}\left(S^{\prime}\right)-R_{b}\left(S_{c}\right)\right\|_{b}^{1} \leq\left\|R_{b}(S)-R_{b}\left(S_{c}\right)\right\|_{b}^{1}$ for $a l l b>1$.

\footnotetext{
${ }^{31}$ Such a Hamiltonian necessarily exists according to Haller and Kennedy (1996).

${ }^{32}$ Note that this condition is possibly stronger than Yin (2011)'s result shows, in that we start in "the other direction"-from a bound on the renormalized Hamiltonian.
} 
Condition 3. Given an arbitrary linearization*-adequate neighborhood $B_{\epsilon}\left(S^{*}\right)$, there exists an open set $V$ in the basin of attraction such that (1) $S^{*} \in V$, and (2) for all surfaces $R_{b}^{n}$ such that $R_{b}^{n} \cap V \neq \emptyset$ and for all $P \in\left(R_{b}^{n} \cap V\right)$, there exists $r \in \mathbb{R}^{+}$such that if $Q \in R_{b}^{n}$, $Q \notin B o A$, and $\|Q-P\|_{b}^{n}<r$, then $Q \in B_{\epsilon}\left(S^{*}\right)$.

Condition 4. For any open set $V$ in the basin of attraction such that $S^{*} \in V$, there exists a linearization*-adequate neighborhood $B_{\epsilon}\left(S^{*}\right)$ such that $\left(\partial B_{\epsilon}\left(S^{*}\right) \cap B o A\right) \subseteq V$; that is, the intersection of the boundary of $B_{\epsilon}\left(S^{*}\right)$ and the basin the attraction is a subset of $V$.

Here, Condition 2 is a condition that, from my observation, practitioners in the field implicitly assume. It holds in the analogy that Wilson and Kogut (1974) present-balls starting closer to the ridge line would stay closer to the ridge, as compared to balls starting further away. Moreover, it would be difficult to imagine that something similar to Condition 2 would be false, since otherwise, we would lack a principled way to connect Hamiltonians close to the nontrivial fixed point back to Hamiltonians close to the critical point on the unrenormalized surface.

Conditions 3 and 4 , on the other hand, are stipulations on the linearization*-adequate neighborhood, which we currently lack adequate understanding about. Nevertheless, I think that these are reasonable conditions to propose. Condition 3 essentially requires that the linearization*-adequate neighborhood is "open." It is stated in a perhaps cumbersome manner because the mathematical structures are only specified on the basin of attraction and on each $R_{b}^{n}$ surface. Condition 4 is reasonable given that the results of linearization* is more accurate the closer the point is to the nontrivial fixed point and to the basin of attraction. This seems to suggest that for any arbitrarily small open set in the basin of attraction containing $S^{*}$, we can have a linearization*-adequate neighborhood whose boundary as it intersects with the basin of attraction sits within the open set.

The above four conditions are sufficient for Conjecture 1.

Proposition 2. Suppose that Conditions 1, 2, 3 and 4 all hold for a physical system $\mathcal{S}$. Conjecture 1 is true for $\mathcal{S}$.

Proof. See Appendix B. 
To connect back to the main concern of the paper-the role of infinite limits systems in RG explanations-I believe that Proposition 2 provides support for the dispensabilists. The success of an essentialist's position hinges on the explanatory necessity of the convergence property, which is only exhibited by infinite limit systems. However, I have demonstrated above that, under reasonable conditions, not only is the convergence property fully equivalent to the linearization* property in terms of demarcating the universality classes, but the linearization* property turns out to be more explanatory relevant than the convergence property in answering the universality question. Because the linearization* property can be exhibited by large but finite systems, we have a case (b') at work (recall the discussion in Section 2). An essentialist's position loses its force.

Admittedly, there are a few limitations to my argument. For one thing, Condition 1 is motivated by Yin (2011)'s work in the context of the Ising model. It is still unclear whether Condition 1 is true in general. Moreover, as I have briefly mentioned before, there are very little discussions, let alone agreements, about which norms and topologies are appropriate to use on the space of Hamiltonians. It might turn out to be the case that there are other norms that are more appropriate to use than the one Yin (2011) identifies. Though, discussions on these alternatives is outside the scope of this paper. Furthermore, the mathematical structures of the space of Hamiltonians that I stipulate may turn out to be unsuitale in some cases. Finally, the auxiliary conditions, though reasonable, have not been definitively shown to hold. To test the viability and limitations of my argument, a worthwhile future research would be to investigate whether the conditions proposed in this paper are satisfied by simple systems such as the Ising model. However, it is important to note that the conditions proposed are sufficient conditions for Conjecture 1, and they may not be necessary. Hence, there could be weaker conditions that would be nonetheless sufficient for the conjecture, if some of the proposed conditions do not hold.

One might still object that to find the linearization*-adequate neighborhood, we need to first form the idea of a nontrivial fixed point, and for that we need infinite limit systems (Batterman, 2017, and from conversations with Batterman). Infinite limit systems occupy a status of explanatory priority, so to speak. I offer three remarks to this objection. First, I 
don't think using infinite limit systems to find the nontrivial fixed point is the only way to do so. Here is a heuristic description of an alternative way of finding the nontrivial fixed point. We know from Proposition 2 that, under reasonable conditions, Hamiltonians that start infinitesimally close to the critical point would spend a large number of iterations very close to the nontrivial fixed point, and Hamiltonians that start closer would find themselves even closer to the nontrivial fixed point. This means that we should be able to construct a sequence $\left\{S_{k}\right\}$ of finite unrenormalized Hamiltonians such that $\left\{R_{b}^{k}\left(S_{k}\right)\right\}$ converges to the nontrivial fixed point, bypassing the need to invoke critical points in the process.

Secondly, scientific practice offers seeds for doubt regarding the necessity of the exact nontrivial fixed point in forming the linearization*-adequate neighborhood and in the success of the RG method. We can only locate the nontrivial fixed point using iterations of RG transformations of critical points in highly idealized situations. In practice, depending on the averaging technique that one uses during the coarse-graining step, the RG trajectories of the system at its critical point might not converge to the nontrivial fixed point at all. For example, the RG trajectories of Hamiltonians at critical points using the "decimation" technique, by which we ignore the contribution by certain microscopic sites, do not converge to the nontrivial fixed point (Yin, 2011, 3). In this case, the "nontrivial fixed point" that they eventually arrive at might not coincide with the exact nontrivial fixed point. ${ }^{33}$ However, physicists and philosophers still agree that "decimation" is a good averaging technique.

To make a final and related point, we can have other ways to formulate the linearization*_ adequate neighborhood without mentioning the nontrivial fixed point, by either making use of the laboratory results of critical exponents and allow a certain error rate, or using a large but finite system as an "alternative nontrivial fixed point." The latter can be done because when the Hamiltonians flow almost to the nontrivial fixed point, they stay in the vicinity for a large number of iterations. In this case, the "alternative nontrivial fixed point" is not entirely fixed after iterations of RG transformations, but its variation is negligible for a large number of iterations. My three remarks suggest that the inference from linearization*adequate neighborhood to the necessity of nontrivial fixed point, and finally to the necessity

\footnotetext{
${ }^{33}$ The point is also not entirely fixed, though its variation after RG transformation would be small.
} 
of infinite limit systems, is not as airtight as critics might believe. On that ground, I maintain my dispensabilist's view.

\section{Conclusion}

To close, by proposing and motivating the linearization* property, I argue against the essentialists on two grounds. First, I argue that convergence properties unique to infinite limit systems are less explanatorily relevant than linearization* properties of finite systems. Second, I presented heuristic and technical evidence by Wilson and Kogut (1974) and Yin (2011) to motivate and prove a proposition demonstrating that, under reasonable conditions, the explanatory functions served by convergence properties can be retrieved by those served by linearization* property, when it comes to demarcating the universality classes. For these reasons, I adopt a dispensabilist's view. 


\section{Appendix A The Linearization* Process}

Through the linearization* process, we accomplish two main feats: first, we categorize the eigenvectors of the RG transformation matrix into relevant, irrelevant, and marginal eigenvectors, and define a basin of attraction spanned by the irrelevant eigenvectors in which all Hamiltonians flow to the nontrivial fixed point; second, we retrieve the critical exponents that characterize the universality class associated with the nontrivial fixed point. In this appendix, I illustrate how the two main feats are achieved through the linearization* process in detail. Note that this explication should be read in a heuristic way, since discussions about the exact mathematical structures of the space of Hamiltonians are still underway. ${ }^{34}$

Following Goldenfeld (1992) and Simons (1997), we start the linearization* process by taking $S=S^{*}+\delta S$, a point in the vicinity of the nontrivial fixed point. We then linearize the RG transformation on $S$,

$$
R_{b}\left(S^{*}+\delta S\right)=R_{b} S^{*}+R_{b} \delta S=S^{*}+R_{b} \delta S .
$$

For $S_{i}$, the $i$-th parameter of the Hamiltonian, we have

$$
S_{i}=S_{i}^{*}+\sum_{j}\left[R_{b}\right]_{i j} \delta S_{j}
$$

We assume that $\left[R_{b}\right]_{i j}$ is a real-valued symmetric matrix. ${ }^{35}$ The semi-group property of $R_{b}$ gives us the relation

$$
\begin{array}{r}
R_{b} R_{b^{\prime}} O_{i}= \\
\lambda_{i}(b) \lambda_{i}\left(b^{\prime}\right) O_{i}=\lambda_{i}\left(b b^{\prime}\right) O_{i} \\
=R_{b b^{\prime}} O_{i}
\end{array}
$$

where $O_{i}$ is an eigenvector of $R_{b}, R_{b^{\prime}}$, and $R_{b b^{\prime}}$, and $\lambda_{i}(b), \lambda_{i}\left(b^{\prime}\right)$, and $\lambda_{i}\left(b b^{\prime}\right)$ are the respective eigenvalues. Now, we differentiate Equation 1 with respect to $b^{\prime}$,

$$
\lambda_{i}(b) \frac{d \lambda_{i}\left(b^{\prime}\right)}{d b^{\prime}} O_{i}=b \frac{d \lambda_{i}\left(b b^{\prime}\right)}{d b b^{\prime}} O_{i}
$$

We can solve Equation 2 for $\lambda_{i}(b)$ with the initial condition $\lambda_{i}(1)=1$, and obtain

$$
\lambda_{i}(b)=b^{y_{i}}
$$

with $y_{i}$ independent of $b$.

We can expand $\delta S$ in terms of the eigenvectors of $R_{b}$,

$$
\delta S=\sum_{i} g^{i} O_{i}
$$

\footnotetext{
${ }^{34}$ For instance, what Hamiltonian addition means can look very different depending on the mathematical structures specified.

${ }^{35}$ Although in theory $\left[R_{b}\right]_{i j}$ is real but not necessarily symmetric, in practice $\left[R_{b}\right]_{i j}$ is often diagonalizable with real eigenvalues. The treatment of non-symmetric matrix admits minor modifications, which I will not mention in the present paper. See (Goldenfeld, 1992) for detailed discussion.
} 
Therefore,

$$
R_{b}(\delta S)=R_{b} \sum_{i} g^{i} O_{i}=\sum g^{i} \lambda_{i}(b) O_{i}=\sum g^{i} b^{y_{i}} O_{i}=\sum g^{i} O_{i}
$$

We then distinguish the following three cases based on the values of $y_{i}$ :

1. $y_{i}>0$. In this case, $g^{\prime i}$ grows after RG transformations.

2. $y_{i}<0$. In this case, $g^{i}$ shrinks after RG transformations.

3. $y_{i}=0$. In this case, $g^{i}$ does not change after RG transformations.

The importance of this categorization is that after iterations of RG transformations, the components of $\delta S$ along directions $O_{i}$ for which case 1 holds are the only relevant components to drive the Hamiltonian away from the non-trivial fixed point. The components of $\delta S$ in other directions either shrink to 0 , driving the Hamiltonian towards the nontrivial fixed point (as in case 2 ), or stay the same (as in case 3 ). Because of this, we give the corresponding eigenvalues $\lambda_{i}(b)$ and eigenvectors $O_{i}$ the following names:

1. $\lambda_{i}(b)$ and $O_{i}$ are relevant eigenvalues/eigenvectors if $y_{i}>0$.

2. $\lambda_{i}(b)$ and $O_{i}$ are irrelevant eigenvalues/eigenvectors if $y_{i}<0$.

3. $\lambda_{i}(b)$ and $O_{i}$ are marginal eigenvalues/eigenvectors if $y_{i}=0$.

The irrelevant eigenvectors span the basin of attraction. Because $g^{i}$ would shrink to 0 after iterations of RG transformations if $O_{i}$ is an irrelevant eigenvector, we know that Hamiltonians that start in the basin of attraction would eventually flow to the nontrivial fixed point. Hamiltonians that start off the basin of attraction would eventually drive away from the basin of attraction towards a trivial fixed point by dint of relevant eigenvalues.

Through the linearization* process, we can also retrieve the critical exponents. To illustrate that, let's consider a simple system with only one parameter, which can be taken to be the temperature, and consider the critical exponent $v$ in $\xi \sim t^{-v}$. Other critical exponents and for more complex systems can be similarly calculated.

For this simple system, we have $T^{\prime}=R_{b}(T)$ and at the critical temperature $T^{*}, T^{*}=$ $R_{b}\left(T^{*}\right) . T=0$ and $T=\infty$ are the two trivial fixed points. Linearizing in the vicinity of the fixed point, we have

$$
\begin{aligned}
T^{\prime}-T^{*} & =R_{b}(T)-R_{b}\left(T^{*}\right) \simeq \lambda_{i}(b)\left(T-T^{*}\right)+\text { corrections }, \\
\lambda_{t}(b) & =b^{y_{t}}
\end{aligned}
$$

where Equation 5 is from Equation 3. We define the reduced temperature

$$
t=\frac{T-T^{*}}{T^{*}}
$$

then together with Equations 4 and 5 we have

$$
t^{\prime}=t b^{y_{t}}{ }^{36}
$$

${ }^{36}$ Here, $t^{\prime}=\frac{T^{\prime}-T^{*}}{T^{*}}$. 
Iterating the RG transformation $n$ times, we have

$$
t^{n}=\left(b^{y t}\right)^{n} t
$$

Now we consider how the correlation length transforms after $n$ RG transformations. Because $\xi^{\prime}=\frac{\xi}{b}$ after one transformations, after $n$ RG transformations we have

$$
\xi(t)=b^{n} \xi\left(t^{n}\right)=b^{n} \xi\left(\left(b^{y t}\right)^{n} t\right)
$$

We can choose $b$ arbitrary so that it satisfies

$$
b^{n}=\left(\frac{u}{t}\right)^{\frac{1}{y_{t}}},
$$

with $u$ being an arbitrary positive number much larger than unity. Equations 6 and 7 together give us

$$
\xi(t)=\left(u^{-1} t\right)^{-\frac{1}{y_{t}}} \xi(u) \quad \text { as } t \rightarrow 0 .
$$

Because $u$ is much larger than unity, $\xi(u)$ is the correlation length for temperatures well above the critical temperature, where fluctuations are small, we can use standard approximation methods to estimate its value. Therefore, if we compare Equation 8 with the power relation between the correlation length and reduced temperature $\xi \sim|t|^{-v}$, we can read off

$$
v=\frac{1}{y_{t}} .
$$

Finally, we know from Equations 4 and 5 that

$$
y_{t}=\frac{1}{b} \log \lambda_{t}(b) \text {. }
$$

So it suffices to know $R_{b}$, or a good approximation of it, in order to calculate $\lambda_{t}(b), y_{t}$, and hence $v$, a critical exponent. 


\section{Appendix B Proofs of Propositions}

\section{Proposition 1}

Proof. Suppose that a system $\mathcal{S}$ is in the universality class delimited by a nontrivial fixed point $S^{*}$. Given an arbitrary linearization*-adequate neighborhood, from Definition 2 we know that there exists $m \in \mathbb{N}$ such that for any arbitrary natural number $n$, there is a Hamiltonian $S$ with $S \notin B o A$ such that for all Hamiltonians $S^{\prime}$ with $0<\left\|S^{\prime}-S_{c}\right\| \leq\left\|S-S_{c}\right\|$ and $S^{\prime} \notin B o A$, we have $R_{b}^{m+i}(S) \in B_{\epsilon}\left(S^{*}\right)$ for all $0 \leq i<n$ and $i \in \mathbb{N}$. Now fix an arbitrary natural number $n$, we have a Hamiltonian $S$ with $S \notin B o A$ such that for all Hamiltonians $S^{\prime}$ with $0<\left\|S^{\prime}-S_{c}\right\| \leq\left\|S-S_{c}\right\|$ and $S^{\prime} \notin B o A, R_{b}^{m+i}(S) \in B_{\epsilon}\left(S^{*}\right)$ for all $0 \leq i<n$ and $i \in \mathbb{N}$. That is to say, all $S^{\prime}$ exhibits the linearization* property. ${ }^{37}$

\section{Proposition 2}

Proof. Suppose that Conditions 1, 2, 3, and 4 all hold for a physical system $\mathcal{S}$.

$\Longrightarrow$ :

Suppose further that $R_{b}^{n}\left(S_{c}\right) \rightarrow S^{*}$ as $n \rightarrow \infty$.

Choose an arbitrary linearization*-adequate neighborhood $B_{\epsilon}\left(S_{c}\right)$. From Condition 3, there exists an open set $V$ in the basin of attraction such that (1) $S^{*} \in V$, and (2) for all surfaces $R_{b}^{n}$ such that $R_{b}^{n} \cap V \neq \emptyset$ and for all $P \in\left(R_{b}^{n} \cap V\right)$, there exists $r \in \mathbb{R}^{+}$such that $Q \in B_{\epsilon}\left(S^{*}\right)$ for all $Q \in R_{b}^{n}, Q \notin B o A$, and $\|Q-P\|_{b}^{n}<r$.

Because $R_{b}^{n}\left(S_{c}\right) \rightarrow S^{*}$ as $n \rightarrow \infty$, we can find $m \in \mathbb{N}$ such that for all $m^{\prime}>m$, we have $R_{b}^{m^{\prime}}\left(S_{c}\right) \in V$. Now choose an arbitrary natural number $n$. We have $R_{b}^{m+i}\left(S_{c}\right) \in V$ for all $0 \leq i<n$.

For each $i \in \mathbb{N}$ with $0 \leq i<n$, since $R_{b}^{m+i}\left(S_{c}\right) \in\left(R_{b}^{m+i} \cap V\right)$, we have some $r_{i}>0$ such that for all points $Q^{\prime}$ with $Q^{\prime} \in R_{b}^{m+i}, Q^{\prime} \notin B o A$, and $\left\|Q^{\prime}-R_{b}^{m+i}\left(S_{c}\right)\right\|_{b}^{m+i}<r_{i}$, we have $Q^{\prime} \in B_{\epsilon}\left(S^{*}\right)$. From Condition 1 , we know that there is a point $S^{i}$ with $S^{i} \notin B o A$ on the original unrenormalized surface, such that

$$
0<\left\|R_{b^{m+i}}\left(S^{i}\right)-R_{b^{m+i}}\left(S_{c}\right)\right\|_{b^{m+i}}^{1}<r_{i} .
$$

By the semi-group property of the RG transformation, $R_{b} R_{b^{\prime}}(P)=R_{b b^{\prime}}(P)$ for all $P$ in the space of Hamiltonians, Equation 9 is equivalent to

$$
0<\left\|R_{b}^{m+i}\left(S^{i}\right)-R_{b}^{m+i}\left(S_{c}\right)\right\|_{b}^{m+i}<r_{i}{ }^{38}
$$

from which we have $R_{b}^{m+i}\left(S^{i}\right) \in B_{\epsilon}\left(S^{*}\right)$.

Now, let $S$ be an unrenormalized point with $S \notin B o A$ such that $0<\left\|S-S_{c}\right\| \leq\left\|S^{i}-S_{c}\right\|$ for all $i<n$. It follows from Condition 2 that

$$
0<\left\|R_{b^{m+i}}(S)-R_{b^{m+i}}\left(S_{c}\right)\right\|_{b^{m+i}}^{1} \leq\left\|R_{b^{m+i}}\left(S^{i}\right)-R_{b^{m+i}}\left(S_{c}\right)\right\|_{b^{m+i}}^{1}<r_{i},
$$

for all $0 \leq i<n$. By the semi-group property of the RG transformation, Equation 11 is equivalent to

$$
0<\left\|R_{b}^{m+i}(S)-R_{b}^{m+i}\left(S_{c}\right)\right\|_{b}^{m+i}<r_{i},
$$

for all $0 \leq i<n$. Therefore, $R_{b}^{m+i}(S) \in B_{\epsilon}\left(S^{*}\right)$.

\footnotetext{
${ }^{37}$ Note that the structure of the proof can be schematized as $\forall x \exists y \forall z(A) \Rightarrow \forall x \forall z \exists y(A)$.

${ }^{38}$ Note that because of the semi-group property, the $R_{b}^{m+i}$ surface is the same surface as the $R_{b^{m+i}}$ surface, with the same norm.
} 
Similarly, from Condition 2, we have, for all $S^{\prime}$ with $S^{\prime} \notin B o A$ such that $0<\left\|S^{\prime}-S_{c}\right\| \leq$ $\left\|S-S_{c}\right\|$

$$
0<\left\|R_{b^{m+i}}\left(S^{\prime}\right)-R_{b^{m+i}}\left(S_{c}\right)\right\|_{b^{m+i}}^{1} \leq\left\|R_{b^{m+i}}(S)-R_{b^{m+i}}\left(S_{c}\right)\right\|_{b^{m+i}}^{1}<r_{i},
$$

for all $0 \leq i<n$. By the semi-group property of the RG transformation,

$$
0<\left\|R_{b}^{m+i}\left(S^{\prime}\right)-R_{b}^{m+i}\left(S_{c}\right)\right\|_{b}^{m+i}<r_{i}
$$

for all $0 \leq i<n$. Therefore, $R_{b}^{m+i}\left(S^{\prime}\right) \in B_{\epsilon}\left(S^{*}\right)$ for all $S^{\prime}$ with $0<\left\|S^{\prime}-S_{c}\right\| \leq\left\|S-S_{c}\right\|$ and $S^{\prime} \notin B o A$. The system $\mathcal{S}$ is in the universality class delimited by $S^{*}$.

Suppose further that $\mathcal{S}$ is in the universality class delimited by $S^{*}$ under Definition 2.

Given an arbitrary open set $V$ in the basin of attraction such that $S^{*} \in V$. By Condition 4, we have a linearization*-adequate neighborhood $B_{\epsilon}\left(S^{*}\right)$ such that $\left(\partial B_{\epsilon}\left(S^{*}\right) \cap B o A\right) \subseteq V$.

Because $\mathcal{S}$ is in the universality class delimited by $S^{*}$ under Definition 2, there exists $m \in \mathbb{N}$ such that for any arbitrary $n \in \mathbb{N}$, there exists $S$ with $S \notin B o A$ on the unrenormalized surface such that for all $S^{\prime}$ with $0<\left\|S^{\prime}-S_{c}\right\|<\left\|S-S_{c}\right\|$ and $S^{\prime} \notin B o A$ we have $R_{b}^{m+i}\left(S^{\prime}\right) \in B_{\epsilon}\left(S^{*}\right)$ for all $0 \leq i<n$ and $i \in \mathbb{N}$.

I will first show that $R_{b}^{m^{\prime}}\left(S_{c}\right) \in \partial B_{\epsilon}\left(S^{*}\right)$ for all $m^{\prime}>m$. To do so, we choose any arbitrary $m^{\prime}>m$ and any arbitrary neighborhood $N\left(R_{b}^{m^{\prime}}\left(S_{c}\right), r\right)=\left\{Q \mid\left\|R_{b}^{m^{\prime}}\left(S_{c}\right)-Q\right\|_{b}^{m^{\prime}}<r\right\}$ on the $R_{b}^{m^{\prime}}$ surface. We want to show that $N\left(R_{b}^{m^{\prime}}\left(S_{c}\right), r\right)$ contains at least one point in $B_{\epsilon}\left(S^{*}\right)$, and at least one point not in $B_{\epsilon}\left(S^{*}\right)$. The latter part is immediately satisfied, since $R_{b}^{m^{\prime}}\left(S_{c}\right) \in N\left(R_{b}^{m^{\prime}}\left(S_{c}\right), r\right)$ and $R_{b}^{m^{\prime}}\left(S_{c}\right) \notin B_{\epsilon}\left(S^{*}\right)$ because it is in the basin of attraction. We're left to show that $N\left(R_{b}^{m^{\prime}}\left(S_{c}\right), r\right)$ contains at least one point in $B_{\epsilon}\left(S^{*}\right)$.

From Condition 1, we have a point $S^{\prime \prime}$ with $S^{\prime \prime} \notin B o A$ on the unrenormalized surface such that $0<\left\|R_{b^{m^{\prime}}}\left(S^{\prime \prime}\right)-R_{b^{m^{\prime}}}\left(S_{c}\right)\right\|_{b^{m^{\prime}}}^{1}<r$. By the semi-group property of the RG transformations, we have $\left\|R_{b}^{m^{\prime}}\left(S^{\prime \prime}\right)-R_{b}^{m^{\prime}}\left(S_{c}\right)\right\|_{b}^{m^{\prime}}<r$. This means that $R_{b}^{m^{\prime}}\left(S^{\prime \prime}\right) \in N\left(R_{b}^{m^{\prime}}\left(S_{c}\right), r\right)$.

Now choose any $n>\left(m^{\prime}-m\right)$, we know that there exists $S$ with $S \notin B o A$ on the unrenormalized surface such that for all $S^{\prime}$ with $0<\left\|S^{\prime}-S_{c}\right\|<\left\|S-S_{c}\right\|$ and $S^{\prime} \notin B o A$ we have $R_{b}^{m+i}\left(S^{\prime}\right) \in B_{\epsilon}\left(S^{*}\right)$ for all $0 \leq i<n$ and $i \in \mathbb{N}$, and in particular, $R_{b}^{m^{\prime}}\left(S^{\prime}\right) \in B_{\epsilon}\left(S^{*}\right)$.

If $\left\|S^{\prime \prime}-S_{c}\right\|<\left\|S-S_{c}\right\|$, then from the above discussion, we have $R_{b}^{m^{\prime}}\left(S^{\prime \prime}\right) \in B_{\epsilon}\left(S^{*}\right)$.

If $\left\|S^{\prime \prime}-S_{c}\right\| \geq\left\|S-S_{c}\right\|$, then for all $S^{\prime}$ with $0<\left\|S^{\prime}-S_{c}\right\|<\left\|S-S_{c}\right\| \leq\left\|S^{\prime \prime}-S_{c}\right\|$, we have $\left\|R_{b^{m^{\prime}}}\left(S^{\prime}\right)-R_{b^{m^{\prime}}}\left(S_{c}\right)\right\|_{b^{m^{\prime}}}^{1}<\left\|R_{b^{m^{\prime}}}\left(S^{\prime \prime}\right)-R_{b^{m^{\prime}}}\left(S_{c}\right)\right\|_{b^{m^{\prime}}}^{1}<r$, by Condition 2. By the semi-group property of the RG transformations, we have $\left\|R_{b}^{m^{\prime}}\left(S^{\prime}\right)-R_{b}^{m^{\prime}}\left(S_{c}\right)\right\|_{b}^{m^{\prime}}<r$. Therefore, $R_{b}^{m^{\prime}}\left(S^{\prime}\right) \in B_{\epsilon}\left(S^{*}\right)$ and $R_{b}^{m^{\prime}}\left(S^{\prime}\right) \in N\left(R_{b}^{m^{\prime}}\left(S_{c}\right), r\right)$.

In both cases above, $N\left(R_{b}^{m^{\prime}}\left(S_{c}\right), r\right)$ contains at least one point in $B_{\epsilon}\left(S^{*}\right)$. Therefore, $R_{b}^{m^{\prime}}\left(S_{c}\right) \in \partial B_{\epsilon}\left(S^{*}\right)$.

It is easy to show that $R_{b}^{m^{\prime}}\left(S_{c}\right)$ is in the basin of attraction, because $S_{c}$ represents the system at its critical point. Therefore, $R_{b}^{m^{\prime}}\left(S_{c}\right) \in\left(\partial B_{\epsilon}\left(S^{*}\right) \cap B o A\right) \subseteq V$ for all $m^{\prime}>m$. Because $V$ is arbitrary, we have $R_{b}^{n}\left(S_{c}\right) \rightarrow S^{*}$ as $n \rightarrow \infty$. 


\section{Appendix C Glossary of Technical Terms}

- Space of Hamiltonians: the space of "Hamiltonians" strictly speaking is a misnomer. In fact, it's the space of coupling constants that serve as parameters in Hamiltonians of some generic form (see, e.g. Palacios (2019)). Every point in the space of Hamiltonians represent a set of values for the coupling constants, and the set of values then correspond to a Hamiltonian.

- $\mathcal{S}$ : this refers to physical systems such as the water-steam system. The system $\mathcal{S}$ can be represented by points on the unrenormalized surface.

- $S, S^{\prime}, S^{\prime \prime}$ : these refer to points on the unrenormalized surface that represent the physical system $\mathcal{S}$.

- $R_{b}^{n}(S)$ : this refers to a point on the space of Hamiltonians which is the result of the $n$-th iteration of the RG transformation of length $b$ applied to the point $S$.

- The $R_{b}^{n}$ surface: this refers to a surface in the space of Hamiltonians that is the codomain of $R_{b}^{n}(S)$, for all $S$ on the unrenormalized surface.

- $S^{*}$ : this is a non-trivial fixed point.

- $S_{c}$ : this is the Hamiltonian on the unrenormalized surface that represents the system $\mathcal{S}$ at its critical point.

- $P, Q, Q^{\prime}$ : these are points in the space of Hamiltonians, not necessarily representing any system.

- Basin of Attraction (BoA): the basin of attraction is a surface in the space of Hamiltonians that is spanned by the irrelevant parameters. $S^{*}$ and $S_{c}$ are both in the basin of attraction. Among other things, the correlation length for points on the basin of attraction is infinite.

- $B_{\epsilon}\left(S^{*}\right)$ : this is the linearization*-adequate neighborhood around the nontrivial fixed point $S^{*}$. 


\section{References}

Baron, S. (2016). The explanatory dispensability of idealizations. Synthese 193(2), 365-386.

Batterman, R. (2015). Autonomy and scales. In Why More is Different, pp. 115-135. Springer.

Batterman, R. W. (2000). Multiple realizability and universality. The British Journal for the Philosophy of Science 51(1), 115-145.

Batterman, R. W. (2001). The devil in the details: Asymptotic reasoning in explanation, reduction, and emergence. Oxford University Press.

Batterman, R. W. (2005). Critical phenomena and breaking drops: Infinite idealizations in physics. Studies In History and Philosophy of Science Part B: Studies In History and Philosophy of Modern Physics 36(2), 225-244.

Batterman, R. W. (2017). Philosophical implications of kadanoff's work on the renormalization group. Journal of Statistical Physics 167(3-4), 559-574.

Batterman, R. W. (2018). Autonomy of theories: An explanatory problem. Noûs 52(4), 858-873.

Batterman, R. W. (2019, 02). Universality and RG Explanations. Perspectives on Science $27(1), 26-47$.

Batterman, R. W. and C. C. Rice (2014). Minimal model explanations. Philosophy of Science 81(3), 349-376.

Bokulich, A. (2008). Reexamining the Quantum-Classical Relation: Beyond Reductionism and Pluralism. Cambridge University Press.

Bokulich, A. (2012). Distinguishing explanatory from nonexplanatory fictions. Philosophy of Science 79(5), 725-737.

Butterfield, J. (2011). Less is different: emergence and reduction reconciled. Foundations of Physics 41(6), 1065-1135.

Callender, C. (2001). Taking thermodynamics too seriously. Studies in History and Philosophy of Science Part B: Studies in History and Philosophy of Modern Physics 32(4), 539-553.

Cardy, J. (1996). Scaling and renormalization in statistical physics, Volume 5. Cambridge university press.

Craver, C. F. (2006). When mechanistic models explain. Synthese 153(3), 355-376.

Earman, J. (2008). Superselection rules for philosophers. Erkenntnis 69(3), 377-414.

Fletcher, S. C., P. Palacios, L. Ruetsche, and E. Shech (2019). Infinite idealizations in science: an introduction. Synthese 196(5), 1657-1669.

Goldenfeld, N. (1992). Lectures on phase transitions and the renormalization group.

Haller, K. and T. Kennedy (1996). Absence of renormalization group pathologies near the critical temperature. two examples. Journal of statistical physics 85(5), 607-637. 
Hempel, C. G. and P. Oppenheim (1948). Studies in the logic of explanation. Philosophy of science 15(2), 135-175.

Kadanoff, L. (1971). Critical behavior, universality and scaling in critical phenomena.

Kadanoff, L. P. (2000). Statistical physics: statics, dynamics and renormalization. World Scientific Publishing Co Inc.

Kadanoff, L. P. (2010). Theories of matter: infinities and renormalization. arXiv preprint arXiv:1002.2985.

Khalifa, K., G. Doble, and J. Millson (2020). Counterfactuals and explanatory pluralism. The British Journal for the Philosophy of Science 71(4), 1439-1460.

Mainwood, P. (2006). Is More Different? Emergent Properties in Physics. Ph. D. thesis, University of Oxford.

Morrison, M. (2015). Why is more different? In Why More Is Different, pp. 91-114. Springer.

Norton, J. D. (2012). Approximation and idealization: Why the difference matters. Philosophy of Science 79(2), 207-232.

Palacios, P. (2019). Phase transitions: A challenge for intertheoretic reduction? Philosophy of Science 86(4), 612-640.

Palacios, P. and G. Valente (2021). The paradox of infinite limits: a realist response. In Contemporary scientific realism. Oxford University Press.

Reutlinger, A. (2014). Why is there universal macrobehavior? renormalization group explanation as noncausal explanation. Philosophy of Science 81(5), 1157-1170.

Shech, E. (2013). What is the paradox of phase transitions? Philosophy of Science 80(5), 1170-1181.

Shech, E. (2015). Two approaches to fractional statistics in the quantum hall effect: Idealizations and the curious case of the anyon. Foundations of Physics 45(9), 1063-1100.

Shech, E. (2018). Infinite idealizations in physics. Philosophy Compass 13(9), e12514.

Simons, B. (1997). Phase transitions and collective phenomena. Lecture Notes, available at http://www.tcm.phy.cam.ac.uk/ bds10/phase.html.

Sklar, L. (2000). Theory and Truth: Philosophical Critique Within Foundational Science. Oxford University Press.

Strevens, M. (2008). Depth: An account of scientific explanation. Harvard University Press.

Wilson, K. G. (1983, Jul). The renormalization group and critical phenomena. Rev. Mod. Phys. 55, 583-600.

Wilson, K. G. and J. Kogut (1974). The renormalization group and the $\epsilon$ expansion. Physics Reports 12(2), 75 - 199.

Woodward, J. (2005). Making things happen: A theory of causal explanation. Oxford university press. 
Yin, M. (2011). Renormalization group transformations near the critical point: Some rigorous results. Journal of Mathematical Physics 52(11), 113507. 